\title{
Direct Experimental Comparison of Krypton and Xenon Discharge Properties in the Magnetic Nozzle of a Helicon Plasma Source
}

Alfio E. Vinci ${ }^{1, a)}$ and Stéphane Mazouffre ${ }^{1, b)}$

Institut de Combustion, Aérothermique, Réactivité et Environnement, Centre National de la Recherche Scientifique, $1 C$ Avenue de la Recherche Scientifique, 45071 Orléans

(Dated: 9 February 2021)

Helicon plasma sources currently represent an active field of research in the domain of low-temperature plasmas due to several interesting characteristics for in-space propulsion applications. This work reports direct comparison of krypton and xenon plasma properties through spatially resolved measurements performed in the near-field plume of a subkilowatt-class $13.56 \mathrm{MHz}$ Helicon source. The set of developed and employed intrusive diagnostics is comprehensively described. The magnetic components of the plasma rf field are inferred using a 3-D B-dot probe along the reactor axial direction with and without the externally applied DC magnetic field. Plasma floating potential fluctuations are measured using a capacitive probe to draw design requirements for a rf-compensated Langmuir probe. The latter is used to perform axial and radial measurements of plasma density, electron temperature and plasma potential. A 4-grids Retarding Potential Analyzer is used to infer the ion kinetic energy downstream the source exit. The effect of magnetic field magnitude on ions and electrons properties is especially investigated.

\section{INTRODUCTION}

The research performed on helicon plasma (HP) discharges ${ }^{1-6}$ has recently become subject of growing interest among the electric space propulsion community ${ }^{7-17}$. These devices feature several attractive characteristics for propulsion purposes, namely a relatively simple design, no need for a neutralizer, expected long lifetime, easy use of alternative propellants and high plasma densities (typically $>10^{17} \mathrm{~m}^{-3}$ ) over a wide range of sizes and input powers. Although still at an early stage ${ }^{18}$, promising performance measurements have been recently reported ${ }^{7,16}$. As a result, HP thrusters have been proposed as an innovative alternative worth to be investigated to enrich the set of already available technologies for use on small platforms ${ }^{19,20}$.

HP sources comprise three main components: $i$ ) a dielectric tube as plasma discharge region, ii) a radiofrequency (rf) emitting antenna for gas ionization and iii) an axially directed DC magnetic field for plasma confinement and expansion throughout its diverging region, i.e. the magnetic nozzle (MN). Power radiated by the antenna is deposited in the electron population as thermal energy by means of helicon wave modes $^{21}$ and Trivelpiece-Gould wave modes ${ }^{22}$. Energy is then spent for plasma production and plasma transport phenomena, such as ambipolar electric field, resulting in axial supersonic acceleration in the MN region ${ }^{23,24}$.

Most of fundamental studies involving HP sources have been employing argon as working gas because of the main interest in plasma processing applications. Likewise, more recent studies focused on space propulsion have equally employed argon propellant $t^{25-27}$. However, it is expected that the use of krypton or xenon in electric propulsion systems would enhance thrust, thrust density and thrust efficiency due to higher ion mass and larger ionization cross section. Thus

a)Electronic mail: alfio.vinci@cnrs-orleans.fr.

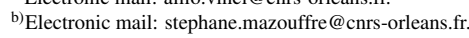

far, only a few works have been reported on HP discharges using either krypton ${ }^{16,28}$ or xenon ${ }^{29-31}$. Nevertheless, it is imperative to extensively characterize the plasma generated using these gases for progressing in terms of maturity and competitiveness of HP thrusters.

This paper analyzes measurements of a variety of plasma properties performed using krypton and xenon gases in the MN region that extends upstream the exit plane. These activities are intended to better understand the physics of such rf plasma sources and to provide a comprehensive dataset for comparison with numerical simulations and for optimization of thruster design and performance. In Section II a detailed description of the HP reactor is provided; the set of diagnostics which has been employed to attain ions and electrons properties is reported in Section III. It includes: a 3-D B-dot probe to measure the rf propagating magnetic field components; a capacitive probe (CP) for detection of floating potential fluctuations; a rf-compensated Langmuir probe (LP) for characterization of plasma density, electron temperature and plasma potential; a Retarding Potential Analyzer (RPA) to assess ions energy. Eventually, in Section IV the collection of measurements is presented and discussed. Conclusions are drawn in Section V.

\section{HELICON PLASMA SOURCE}

The HP source employed in this work is schematically depicted in Fig. 1. It comprises a vertically oriented quartz tube with $\phi=9 \mathrm{~cm}$ inner diameter and $L=58 \mathrm{~cm}$ length, whose open exit defines the origin of the system axial coordinate $z$. The top aperture is equipped with an off-axis gas injector, while the bottom part is connected to a $30 \mathrm{~cm}$ in inner diameter and $50 \mathrm{~cm}$ in length expansion chamber. One lateral side of the chamber is connected to a turbomoleular/primary pumping system providing an ultimate base pressure in the order of $10^{-5} \mathrm{mbar}$. Typical values of pressure during operation lay in the $10^{-3}$ mbar range.

$\mathrm{RF}$ power is generated by a $1 \mathrm{~kW}$ class power supply at 


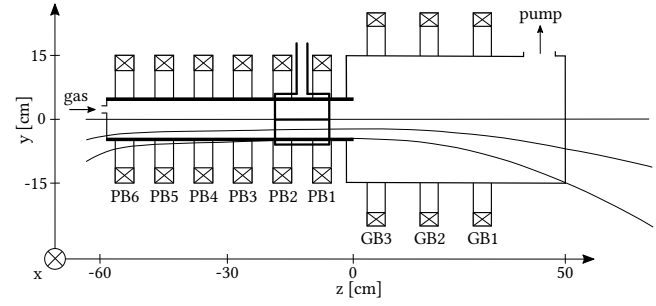

FIG. 1. Helicon Plasma Reactor Schematics. The $z=0$ position refers to the tube outlet, $y=0$ refers to the reactor axis.

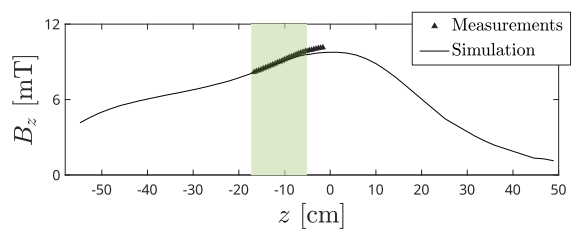

FIG. 2. Externally applied magnetic field profile. The shaded area indicates the antenna location.

13.56 MHz and fed into a copper-made double-saddle antenna through a $2 \mathrm{~kW}$ custom $\pi$-type matching network. The antenna is $12 \mathrm{~cm}$ high and its center is located at $z \cong-11 \mathrm{~cm}$. It is designed such that it is directly connected to the matching network to reduce power transfer losses. A rendering of the antenna is provided in Fig. 3.

The HP source features nine electromagnets, also depicted in Fig. 1. The electromagnets surrounding the expansion chamber are named GB and numbered from 1 to 3 , moving upstream. The same progression is applied to those surrounding the quartz tube, named PB from 1 to 6 . Each electromagnet is made up of a $2 \mathrm{~mm}$ diameter copper wire, constituting 510 loops for PB1-6 and 430 loops for GB1-3. Fig. 2 illustrates the measured and simulated intensity of $B_{z}$ obtained by driving a current of 7 A through GB3 and 1 A through PB1-6 and GB2. This arrangement allows a specific magnetic topology with the magnetic throat exactly at $z=0$ with strength of about $10 \mathrm{mT}$. The same qualitative topology applies for three additional scaled-down magnetic profiles with peak values of about 7,5 and $4 \mathrm{mT}$, which are investigated to obtain the results contained in Section IV D.

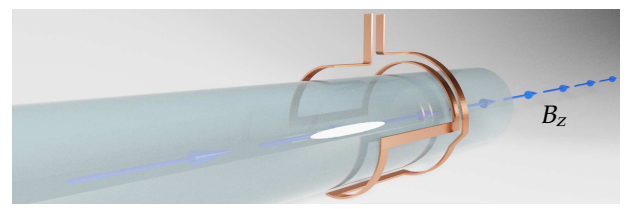

FIG. 3. Rendering of double-saddle antenna wrapped about the discharge tube. Electromagnets and expansion chamber are not shown.

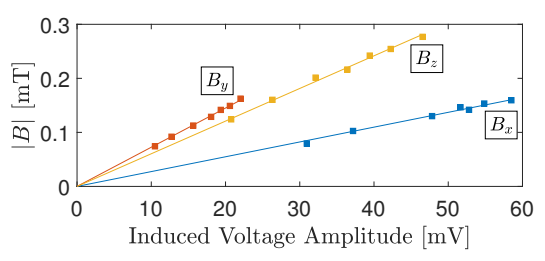

FIG. 4. B-dot probe calibration results.

\section{DIAGNOSTICS}

\section{A. B-dot Probe}

The 3-D B-dot probe developed for this work is inspired by the design reported in Ref. (32) and it consists of: a main body, three orthogonal magnetic pickup coils, a protective cover and a noninductive pickup rejection filtering unit.

The main body is a $4 \mathrm{~mm}$ outer diameter alumina tube which has been hand-shaped to allow winding of the coils. Each coil consists of 3 loops of $0.22 \mathrm{~mm}$ insulated copper wire. The coils assembly is shielded from direct exposure to plasma by using a PEEK cover with $8 \mathrm{~mm}$ outer diameter. Wire leads are first arranged in the twister pair configuration nearby the coils assembly, and finally they are converted into six coaxial cables. These are eventually connected to a 2.5 GS/s digital oscilloscope through the noninductive pickup rejection filtering unit ${ }^{33,34}$. It consists of one sub-unit per coil, each of which features a Coilcraft SWB2010-PCL 1:1 centertapped transformer and a Faraday shield.

The magnetic sensitivity of the probe is absolutely calibrated using two magnetic coils in the Helmholtz configuration with a radius of $60 \mathrm{~mm}$ and 100 loops. Power at $13.56 \mathrm{MHz}$ between $5 \mathrm{~W}$ and $20 \mathrm{~W}$ is fed into the coils connected in parallel to a $50 \Omega$ resistor to the ground. Current flowing in the coils is measured using a Pearson Current Monitor 2878, post-processed using a Savitzky-Golay filter and used to compute the rf magnetic field amplitude, which is finally related to the measured induced voltage in the probe coil by a linear fit. The resulting calibration curves are reported in Fig. 4.

\section{B. Capacitive Probe}

The capacitive probe developed for this work is based on the design reported in Ref. (35). It comprises an exposed electrode made of stainless steel and an inner electrode connected to a coaxial cable. An exposed insulator layers made of PEEK is interposed between the two electrodes. The coaxial cable is carried into the plasma inside a $4 \mathrm{~mm}$ alumina tube which also provides structural support to the electrodes assembly. The probe has a cylindrical geometry with an outermost diameter of $10 \mathrm{~mm}$.

For calibration, an aluminum foil envelopes the primary 


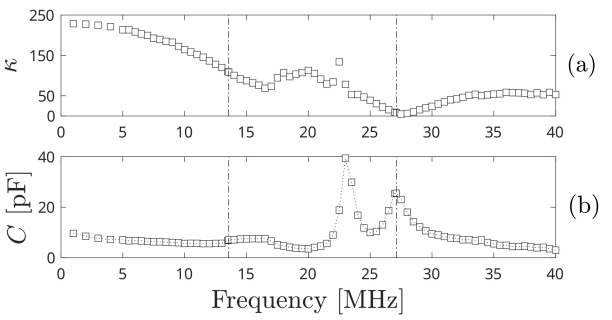

FIG. 5. Capacitive probe (a) calibration coefficient and (b) capacitance vs. frequency.

electrode and the PEEK layer. A $10 \mathrm{~V}$ amplitude sine wave is generated at 1 to $40 \mathrm{MHz}$ and applied to the aluminum foil. The probe is connected to a $2.5 \mathrm{GS} / \mathrm{s}$ digital oscilloscope to acquire the measured voltage and retrieve the calibration factor $\kappa$ reported in Fig. 5(a) as function of the input frequency. Probe capacitance $C$ is inferred by measuring the current flowing in the probe cable using a Pearson Current Monitor 2878 Results are reported in Fig. 5(b). In both plots, dash-dotted lines indicate $13.56 \mathrm{MHz}$ and second harmonic. Values for $\kappa$ and $C$ at the third harmonic are approximated to those at $40 \mathrm{MHz}$ due to setup limitations. Note that the probe exhibits a non-ideal behavior in the $\sim 17-35 \mathrm{MHz}$ range.

\section{RF-compensated Langmuir Probe}

The rf-compensated LP is depicted in Fig. 6. Its design is based on the results and guidelines reported in Ref. (36-38). The probe tip is a $0.38 \mathrm{~mm}$ tungsten wire with $5 \mathrm{~mm}$ length A $1 \mathrm{~mm}$ outer diameter alumina tube insulates the tip from the rf compensating electrode made of stainless steel. The back end of the compensating electrode is soldered to a $1 \mathrm{nF}$ axial capacitor. The probe tip and the small capacitor are together connected to a series of three chokes, each of which self-resonates at one of the first three harmonics. The employed chokes are: Bourns 78F270J-RC for the fundamental harmonic, API Delevan 1025-60K for the second one and Bourns 9230-52-RC for the third one. The whole assembly is encapsulated in a $6 \mathrm{~mm}$ outer diameter Pyrex tube which also accommodates the coaxial cable for probe biasing and current measuring.

Characterization of the chokes is a crucial aspect of probe development as they are typically manufactured to satisfy a requirement on the inductance value but not on the selfresonating frequency. Therefore, an Agilent 4264A precision analyzer has been used to characterize the choke for the funda-

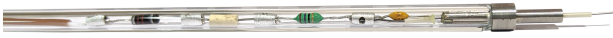

FIG. 6. rf-compensated Langmuir Probe.

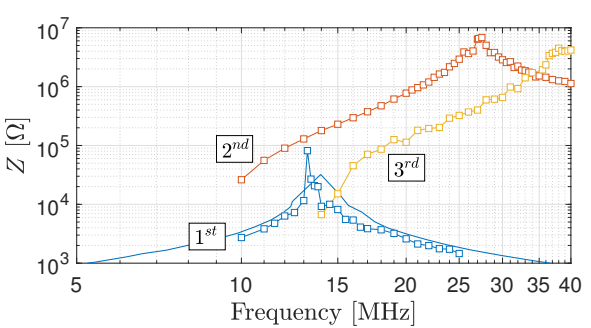

FIG. 7. Impedance of $1^{\text {st }}, 2^{\text {nd }}$ and $3^{\text {rd }}$ harmonic chokes.

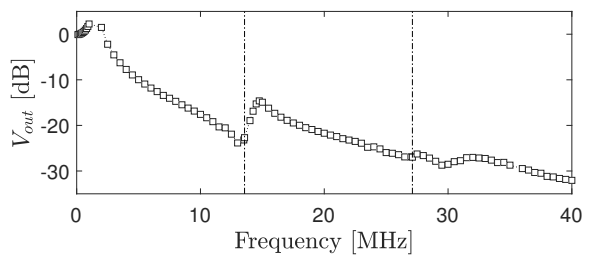

FIG. 8. rf-compensated LP characterization.

mental harmonic. It also allowed validation of a more easily accessible method hereafter described which has been used to characterize the other two chokes. A $10 \mathrm{~V}$ amplitude sine wave is fed to one lead of the testing choke. The other lead is connected to a resistor ( $46.52 \mathrm{k} \Omega$ is used in this setup) and the series circuit closes to the ground. Input frequency is changed while the voltage drop across the resistor is measured and used to calculate the current flowing in the circuit. The frequency at which the current is minimum is the self-resonating frequency of the choke. The voltage drop across the choke is maximum at resonance. Impedance is then computed using Ohm's law. Chokes characterization results are reported in

Fig. 7. The blue solid line represents the impedance profile obtained using the Agilent 4264A precision analyzer and blue squares are obtained with the aforementioned method, showing good agreement. The theory illustrated in Ref. 36 allows stating that this configuration of chokes series and compensating electrode enables proper rf compensation for plasma potential fluctuations up to $99.5 \mathrm{~V}$ in krypton and $85.1 \mathrm{~V}$ in xenon. The whole probe is characterized by applying a $10 \mathrm{~V}$ amplitude sine wave to the tip at different frequencies. The probe output is illustrated in Fig. 8 in dB units, showing local minima for the desired harmonics (dash-dotted vertical lines indicate first and second harmonics).

\section{Retarding Potential Analyzer}

The RPA, also known as Retarding Field Electrostatic Analyzer (RFEA), used in this work consists of four mesh grids with $0.4 \mathrm{~mm}$ mesh size: the entrance grid $(\mathrm{G} 1)$, the electron 
repeller grid (G2), the ion filtering grid (G3) and the secondary electron repeller grid (G4). A collector (C) is placed downstream the grids assembly to enable ion current measurement. The whole assembly is encapsulated in an aluminum body with $4.5 \mathrm{~cm}$ outer diameter and $1 \mathrm{~cm}$ entrance orifice. The gap between all the elements is kept constant at $1 \mathrm{~mm}$. In principle, this is not compatible with the expected value of Debye length in the bulk of the plasma (in the order of $10^{-2} \mathrm{~mm}$ ), implying a requirement on the grid spacing to be smaller than about $0.15 \mathrm{~mm}$ to avoid space charge limited flow ${ }^{39}$. Such a grid spacing is technologically difficult to achieve. Hence, to reduce the plasma flux entering the probe, i.e. the plasma density inside the probe itself, an additional grid (G0) is placed in contact with the outer body. This allowed to significantly increase the quality of the measured I-V signal. The grid polarization scheme with respect to ground is: $\mathrm{G} 0$ at $0 \mathrm{~V} ; \mathrm{G} 1$ at $0 \mathrm{~V} ; \mathrm{G} 2$ at $-60 \mathrm{~V} ; \mathrm{G} 3$ swept from $-20 \mathrm{~V}$ to $100 \mathrm{~V}$ with $0.2 \mathrm{~V}$ step size; $\mathrm{G} 4$ at $-30 ; \mathrm{C}$ at $-3 \mathrm{~V}$.

\section{EXPERIMENTAL RESULTS AND DISCUSSION}

All the spatially-resolved measurements presented hereafter are obtained by feeding $750 \mathrm{~W}$ rf power to the antenna Reflected power is below $2.5 \%$ for all operating conditions. Krypton and xenon are injected at $1 \mathrm{mg} / \mathrm{s}$ and $0.2 \mathrm{mg} / \mathrm{s}$, respectively. Assuming the two gases have the same molar vol-
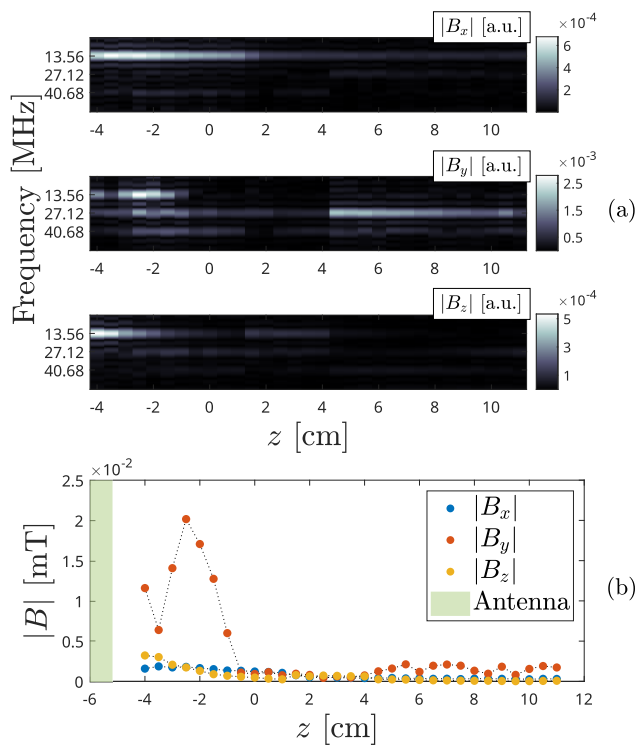

FIG. 9. B-dot probe measurements with $P_{I N}=750 \mathrm{~W}, \dot{m}=1 \mathrm{mg} / \mathrm{s}$ $\mathrm{Kr}$ and no external B field. Spatially-resolved (a) frequency spectrum and (b) first harmonic amplitude. ume, the neutral density is about 8 times larger for krypton. These mass flow rate values are implicitly assumed in the following when not specified.

The diagnostics presented in Section III is manually actuated using a single-axis translation stage with a resolution of $10 \mu \mathrm{m}$ and $150 \mathrm{~mm}$ stroke. Probes are individually employed and displaced with a step size of $5 \pm 0.05 \mathrm{~mm}$. Probe alignment is ensured by use of a cross-line laser pendulum.

\section{A. RF Propagating Magnetic Field}

The B-dot probe is mounted off axis at $r=30 \mathrm{~mm}$ and displaced axially. It is mounted in such a way that the vector defining its $y$-coil, i.e. the coil probing the $B_{y}$ component of the propagating field, is aligned with the antenna feeding lines. The notation is consistent with the reference frame illustrated in Fig. 1. It should be noted that there is an ambiguity of 180 in the phase of the recorded signals, yet it is of no relevance for this study.

At each axial location of the probe, the waveform of the induced voltage on the three coils is acquired using a $2.5 \mathrm{GS} / \mathrm{s}$ digital oscilloscope. Noise reduction on the signal is attained using a Savitzky-Golay filter. Fast Fourier Transform (FFT) is then performed to infer the spatially-resolved frequency spectrum for $B_{x}, B_{y}$ and $B_{z}$. Since the probe is absolutely calibrated at $13.56 \mathrm{MHz}$ only, the FFT component at this frequency is
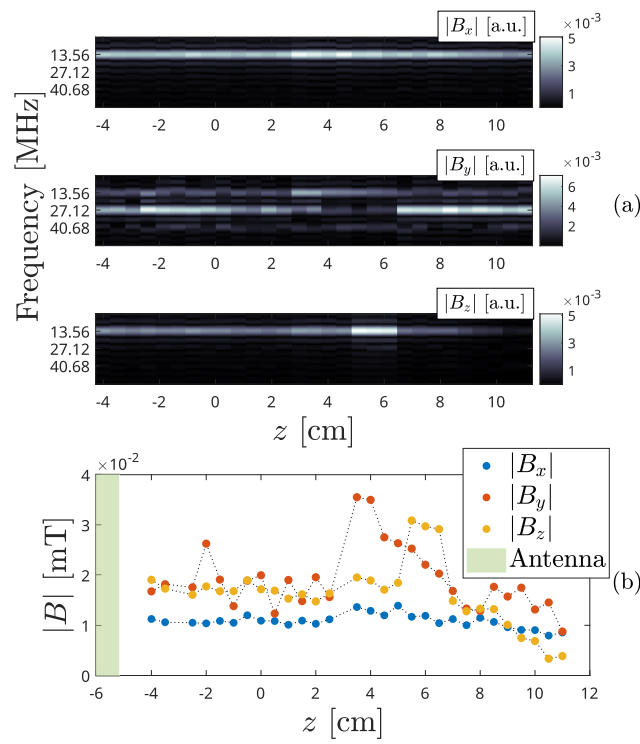

FIG. 10. B-dot probe measurements with $P_{I N}=750 \mathrm{~W}, \dot{m}=1 \mathrm{mg} / \mathrm{s}$ $\mathrm{Kr}$ and $10 \mathrm{mT}$ peak value of the external B field. Spatially-resolved (a) frequency spectrum and (b) first harmonic amplitude. 

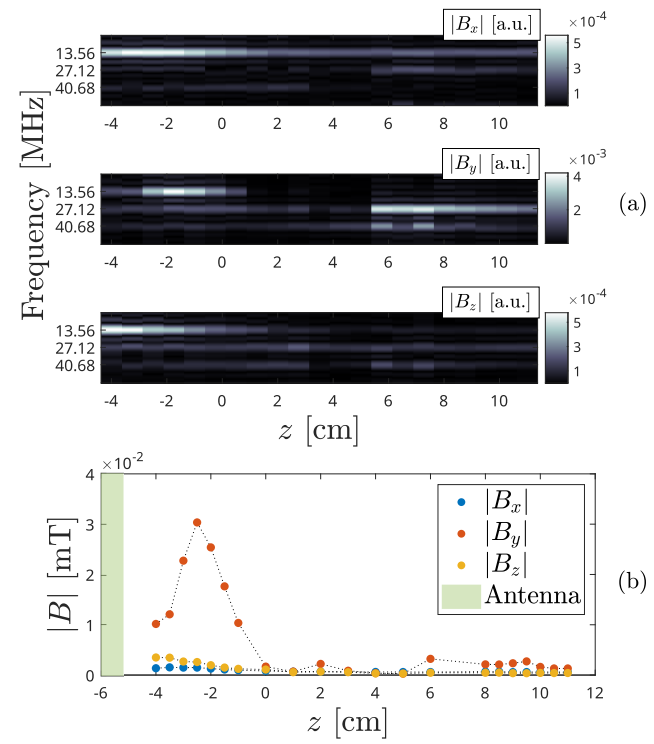

FIG. 11. B-dot probe measurements with $P_{I N}=750 \mathrm{~W}, \dot{m}=0.2 \mathrm{mg} / \mathrm{s}$ $\mathrm{Xe}$ and no external B field applied. Spatially-resolved (a) frequency spectrum and (b) first harmonic amplitude.

extracted to quantitatively evaluate the $\mathrm{rf} B$ field amplitude.

Results using krypton as propellant are reported in Fig. 9 and Fig. 10. The measurements of Fig. 9 are obtained without applying any external DC magnetic field, while those in Fig. 10 result from the application of the magnetic profile depicted in Fig. 2. Similar qualitative results are obtained using xenon as propellant and are reported in Fig. 11 for the $B=0$ case and in Fig. 12 for the externally applied magnetic field case.

It is immediately evident from Fig. 9 and Fig. 11 that no electromagnetic wave propagates in the $B=0$ case, as expected. Indeed, visual inspection of the reactor operating in this condition strongly suggests an inductively-coupled regime, with a very bright plasma just below the antenna and very low light emission elsewhere, either using krypton or xenon. Differently, when the external magnetic field is applied, the plasma column appears quite uniform over a length about ten times larger than the antenna length, suggesting higher power absorption and transport downstream, typical of helicon mode plasmas ${ }^{4,5}$. In the $B=0$ configuration, larger amplitude of $B_{y}$ is recorded in the upstream region of the axial scan, see Fig. 9(b) and Fig. 11(b), suggesting it is related to the field radiated by the antenna ${ }^{40}$. By comparing Fig. 9(a) and Fig. 10(a), as well as Fig. 11(a) and Fig. 12(a), it is noted that the presence of higher harmonics in $B_{x}$ and $B_{z}$ components is reduced when the external magnetic field is applied, while they are anyhow present in the $B_{y}$ component
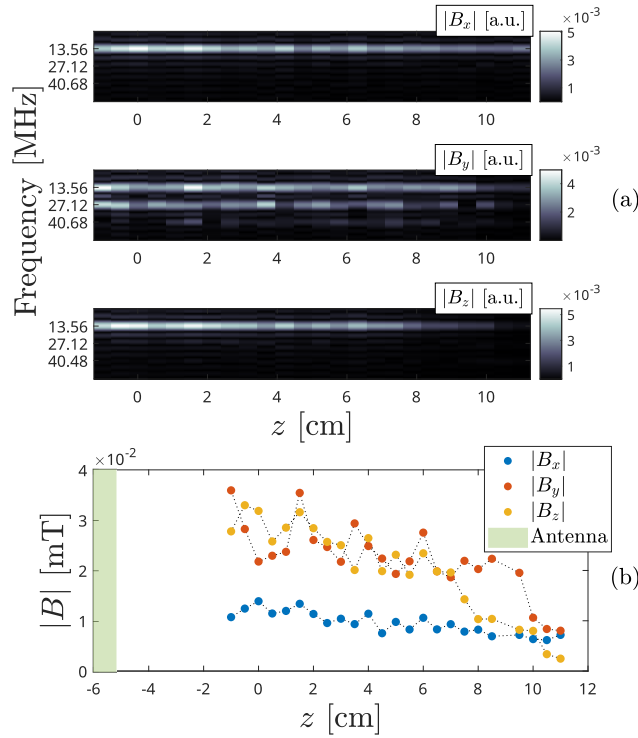

FIG. 12. B-dot probe measurements with $P_{I N}=750 \mathrm{~W}, \dot{m}=0.2 \mathrm{mg} / \mathrm{s}$ $\mathrm{Xe}$ and $10 \mathrm{mT}$ peak value of the external B field. Spatially-resolved (a) frequency spectrum and (b) first harmonic amplitude.

Higher order harmonics cannot be attributed to the rf power supply, which is certified to carry harmonics below $-40 \mathrm{dBc}$. As their presence is susceptible to the external magnetic field, it is reasonable to consider they are related to some physical phenomenon. However, it is not possible to accurately quantify the influence of each harmonic due to the lack of calibration factors at frequencies different from the fundamental one. Furthermore, with reference to Fig. 10(b) and 12(b), an important amplitude decay can be observed for $B_{z}$ approximately for $z>7 \mathrm{~cm}$. This could be explained by observing that the applied magnetic field starts weakening rapidly at $z \cong 7 \mathrm{~cm}$, cf. Fig. 2. Also, it shall be shown in Section IV C that the plasma density $n_{p}$ monotonically increases for $z>0$. Using the 0-D dispersion relation for helicon waves ${ }^{4,41}$,

$$
k k_{z}=e \mu_{0} \omega \frac{n_{p}}{B_{0}},
$$

where $k$ and $k_{z}$ are respectively the total and axial wavenumbers, $n_{p}$ is the plasma density, $B_{0}$ is the assumed uniform external magnetic field and the rest is convential, it can be qualitatively stated that as the ratio $n_{p} / B_{0}$ increases, the condition for wave propagation is met only for higher order axial wavenumbers. These are not necessarily efficiently coupled with the antenna, therefore helicon waves could not propagate further downstream. However, lack of data at larger axial locations prevents excluding that the amplitude decay of $B_{z}$ is rather due to modulation, which has been previ- 


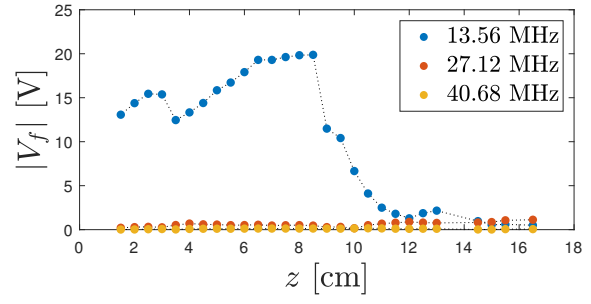

FIG. 13. $V_{f}$ fluctuation amplitude vs. $z \cdot P_{I N}=750 \mathrm{~W}, 1 \mathrm{mg} / \mathrm{s} \mathrm{Kr}$, $r=0 \mathrm{~mm}$ and $10 \mathrm{mT}$ peak value for the B field.

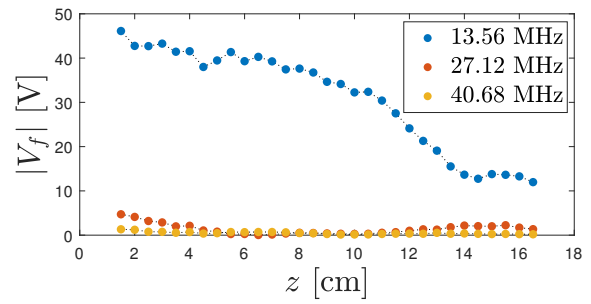

FIG. 14. $V_{f}$ fluctuation amplitude vs. $z . P_{I N}=750 \mathrm{~W}, 1 \mathrm{mg} / \mathrm{s} \mathrm{Kr}$, $r=30 \mathrm{~mm}$ and $10 \mathrm{mT}$ peak value for the B field.

ously observed and attributed to the presence of multiple axial wavenumbers ${ }^{4,21}$

\section{B. Floating Potential Fluctuations}

It is well known that typical plasma potential fluctuations occurring in rf plasmas substantially alter the I-V characteristic of a LP, leading to a poor estimation of plasma properties $^{36-38}$. In order to properly design a passively rfcompensated LP, i.e. chokes selection and compensating electrode sizing ${ }^{36}$, it is important to estimate the amplitude of these fluctuations within the operating condition of interest. To do so, the capacitive probe described in Section III B is immersed in the plasma and displaced axially in a similar fashion to the B-dot probe. Axial scans have been performed at $r=0$ and $r=30 \mathrm{~mm}$ radial locations. At each axial location, the measured voltage is recorded using a $2.5 \mathrm{GS} / \mathrm{s}$ digital oscilloscope. FFT allows isolating the first three harmonics out of the signal which are scaled according to the calibration outcomes. Results obtained using krypton are reported in Fig. 13 and Fig. 14, while those using xenon are in Fig. 15 and Fig. 16

It is clearly evident that fluctuations at the second and third harmonic are weakly present in the spectrum. With reference to Fig. 14 and Fig. 16, larger amplitudes of the oscillating floating potential are recorded off-axis, starting from few tens of volts close to the quartz tube exit and then smoothly decaying to zero downstream. Comparing on-axis profiles us-

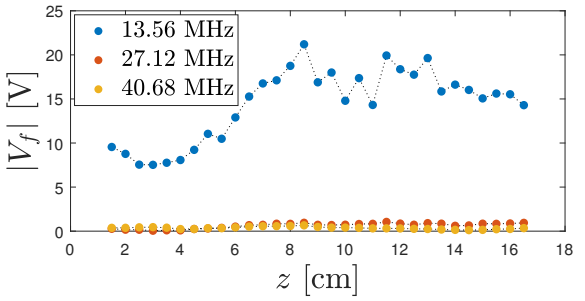

FIG. 15. $V_{f}$ fluctuation amplitude vs. $z \cdot P_{I N}=750 \mathrm{~W}, 0.2 \mathrm{mg} / \mathrm{s} \mathrm{Xe}$, $r=0 \mathrm{~mm}$ and $10 \mathrm{mT}$ peak value for the B field.

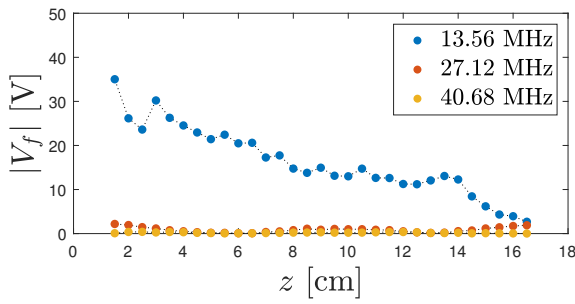

FIG. 16. $V_{f}$ fluctuation amplitude vs. $z \cdot P_{I N}=750 \mathrm{~W}, 0.2 \mathrm{mg} / \mathrm{s} \mathrm{Xe}$, $r=30 \mathrm{~mm}$ and $10 \mathrm{mT}$ peak value for the B field.

ing krypton and xenon, cf. Fig. 13 and Fig. 15, it is evident that substantially different results are obtained. After a shared increase of $\left|V_{f}\right|$, a very sharp edge is present in Fig. 13 at $z=8.5 \mathrm{~cm}$. The reason behind this remains unclear.

Ultimately, it is observed that $V_{f}$ fluctuations amplitude can reach an upper value of about $50 \mathrm{~V}$. This value is taken as requirement in designing the rf-compensated LP described in Section III C.

\section{Plasma Properties}

Characterization of plasma properties has been carried out using the rf-compensated LP described in Section III C. I-V characteristics are obtained by using an automated control unit by Impedans Ltd and afterwards interpreted using the $\mathrm{OML}_{\text {theory }}{ }^{38}$. An example is provided in Fig. 17. Ion current squared is plotted versus probe potential and fitted by a straight line, enabling the evaluation of plasma density $n_{p}$ (assuming local quasi-neutrality). The ion current fit is subtracted to the total current to obtain a better estimation of the electron current. Assuming that the electron population is described by a Maxwell-Boltzmann distribution function, the logarithmic curve of the electron current is fitted by a straight line. Electron temperature $T_{e}$ is deduced from the slope of the linear fit and plasma potential $V_{p}$ is consequently inferred using the electron current equation with the known values of $n_{p}$ and $T_{e}$. 

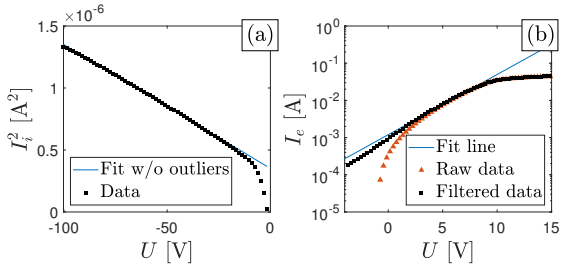

FIG. 17. Typical (a) squared ion current and (b) electron curren vs. probe potential. Curves obtained for $P_{I N}=750 \mathrm{~W}, \dot{m}=1 \mathrm{mg} / \mathrm{s}$ $\mathrm{Kr}, 10 \mathrm{mT}$ peak value of the external $\mathrm{B}$ field.

A statistical analysis is performed to evaluate the uncertainty associated with the assessment of plasma properties. The rf-compensated LP is placed at two axial locations $(z=-5 \mathrm{~cm}$ and $z=10 \mathrm{~cm})$, at each of which twenty I-V characteristics are recorded in different moments of the day, randomly switching between the magnetic configurations investigated in Section IV D. Values for $n_{p}, T_{e}$ and $V_{p}$ are computed at both $z=-5 \mathrm{~cm}$ and $z=10 \mathrm{~cm}$ as previously discussed, together with the associated mean value $\mu$ and standard deviation $\sigma$. The error is then computed as the ratio $\sigma / \mu$. Starting from the errors evaluated at $z=-5 \mathrm{~cm}$ and $z=10 \mathrm{~cm}$, a single representative value is calculated as the average of the two The whole process is repeated for krypton and xenon propellants. Results are reported in Table I. It is shown that $n_{p}, T_{c}$ and $V_{p}$ are measured with an error as high as $\sim 2 \%$ in all cases

Data is collected by displacing the probe radially between $r=0 \mathrm{~mm}$ and $r=30 \mathrm{~mm}$ and axially, both directions with a step size of $5 \mathrm{~mm}$. Seven axial profiles for $n_{p}, T_{e}$ and $V_{p}$ are obtained. By interpolation, contour plots for kypton and xenon are derived and shown in Fig. 18(a)-(d) and Fig. 19(a) (d), respectively. Electron pressure $p_{e}$ is computed using the measured density and temperature. Peak values for $T_{e}$ are about $4.5 \div 5 \mathrm{eV}$ both for krypton and xenon, occurring offaxis and few centimeters downstream the antenna. Then, from $z \cong 0$ on, the temperature decays monotonically. Counterintuitively, $n_{p}$ is peaked downstream where $T_{e}$ is low. It is notable that the same qualitative results have been previously found in numerical ${ }^{42}$ and experimental ${ }^{5}$ studies using completely different setups in terms of scale and input parameters, such as antenna type and associated azimuthal mode numbers, input power frequency, magnetic field strengths and propel-

\begin{tabular}{c|c|c|c|c|c|c} 
& \multicolumn{2}{|c|}{$\sigma_{n_{p}} / \mu_{n_{p}} \times 100$} & \multicolumn{2}{|c|}{$\sigma_{T_{e}} / \mu_{T_{e}} \times 100$} & \multicolumn{2}{|c}{$\sigma_{V_{p}} / \mu_{V_{p}} \times 100$} \\
& $\mathrm{Kr}$ & $\mathrm{Xe}$ & $\mathrm{Kr}$ & $\mathrm{Xe}$ & $\mathrm{Kr}$ & $\mathrm{Xe}$ \\
\hline \hline $4 \mathrm{mT}$ & $0.90 \%$ & $0.64 \%$ & $0.31 \%$ & $0.37 \%$ & $1.05 \%$ & $0.56 \%$ \\
$5 \mathrm{mT}$ & $1.07 \%$ & $0.85 \%$ & $0.59 \%$ & $0.34 \%$ & $1.99 \%$ & $1.17 \%$ \\
$7 \mathrm{mT}$ & $0.76 \%$ & $0.78 \%$ & $0.69 \%$ & $0.51 \%$ & $2.17 \%$ & $0.72 \%$ \\
$10 \mathrm{mT}$ & $1.22 \%$ & $0.49 \%$ & $0.84 \%$ & $0.43 \%$ & $1.41 \%$ & $0.75 \%$ \\
\hline
\end{tabular}

TABLE I. Uncertainty analysis of rf-compensated LP measurements: percentage error of plasma properties for four different magnetic field strengths. lant. It shall be noted that likewise reported in Ref. (5), also in the present case this phenomenon is due to pressure balance. In a simplified 1-D two fluids description of the plasma, the axial component of the electron momentum equation in cylindrical coordinates reads

$$
-n_{p} e E_{z}-j_{\theta_{e}} B_{r}-\frac{\partial p_{e}}{\partial z}=0,
$$

where $p_{e}$ is the electron pressure, $E_{z}$ is the axial electric field, $j_{\theta_{e}}$ is the electron azimuthal current density and $B_{r}$ is the radial component of the applied magnetic field. For simplicity, the momentum transfer collisional term has been neglected. This is justified by observing that the mean free path $\lambda$ is much larger than the source diameter. Values of $\lambda$ are computed using average values for $n_{p}$ and $T_{e}$ from Fig. 18 and Fig. 19 together with the available dataset for the elastic momentum transfer cross section ${ }^{43}$, yielding $\lambda \approx 25 \mathrm{~m}$ for krypton and $\lambda \approx 15 \mathrm{~m}$ for xenon. With reference to Fig. 1 , it can be observed that $B_{r} / B_{z} \ll 1$ in the region where the scan is performed, with typical values of $B_{r} \approx 10^{-6} \div 10^{-7} \mathrm{~T}$. The electron current density shall be shown to be $j_{\theta_{e}} \lesssim 10^{3} \mathrm{~A} / \mathrm{m}^{2}$, cf. Fig. 25 and Fig. 26 later described. Hence, the electrons momentum equation reduces to a balance between the volumetric electric forces term, i.e. $-n_{p} e E_{z}$, and the volumetric pressure forces term, i.e. $-\partial p_{e} / \partial z$. By averaging the measured $n_{p}(r, z), V_{p}(r, z)$ and $p_{e}(r, z)$ over the radial direction, both terms of Eq. 2 can be easily evaluated. Results are reported in Fig. 20(a) for krypton and Fig. 20(b) for xenon. It is shown that pressure gradient and potential gradient are nearly equal and opposite through all the scanned region. Yet, this is more evident for krypton than for xenon, potentially due to the neglected collisional term in Eq. 2. Hence, it is concluded that the density peak localized downstream is due to pressure balance. As the external magnetic field reduces the cross-field electron mobility, a relatively large axial conductivity ensues, which determines an almost constant plasma potential profile. This results in a small pressure gradient for equilibrium reasons, cf. Equation 2. The density peaks downstream because the temperature drops.

It is worth noting that, by knowing the number of neutrals injected in the system per unit time $\dot{N}_{g}=\dot{m} / M_{i}$, where $M_{i}$ is the ion mass, and by taking a tentative value for the average plasma density $\bar{n}_{p}$ accounting for all measured values in Fig. 18(a) and Fig. 19(a), it is possible to further compare the two working gases in terms of local propellant utilization efficiency $\eta_{p}$. It is implicitly assumed that the two gases share the same mean ion charge as $T_{e}$ is relatively low to enable a significant amount of multiply charged particles. Since $\eta_{p} \propto \bar{n}_{p} / N_{g}$, it is computed that $\eta_{p}^{X e} / \eta_{p}^{K r} \approx 5$, suggesting that xenon is much more efficiently ionized, as expected.

\section{Effect of Different Magnetic Strengths}

Plasma properties are also investigated as function of the external magnetic field strength. By tuning the current in the electromagnets, the magnetic profile depicted in Fig. 2 can be scaled down attaining peak values of $7 \mathrm{mT}, 5 \mathrm{mT}$ and $4 \mathrm{mT}$. 

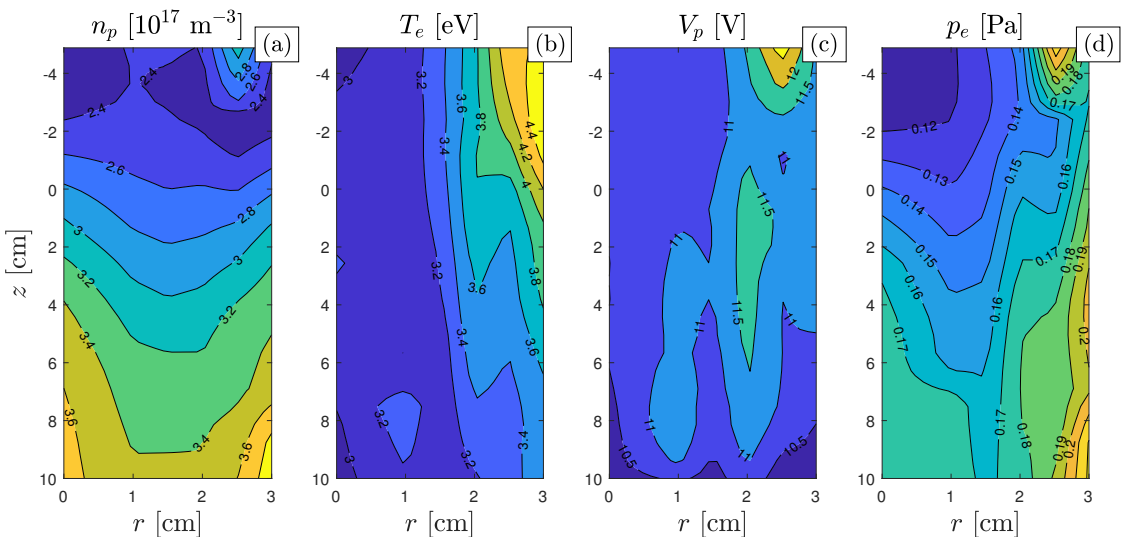

FIG. 18. Contour plot of (a) plasma density, (b) electron temperature, (c) plasma potential, (d) electron pressure. Input parameters are: $P_{I N}=750 \mathrm{~W}, \dot{m}=1 \mathrm{mg} / \mathrm{s} \mathrm{Kr}, 10 \mathrm{mT}$ peak value of the external B field.
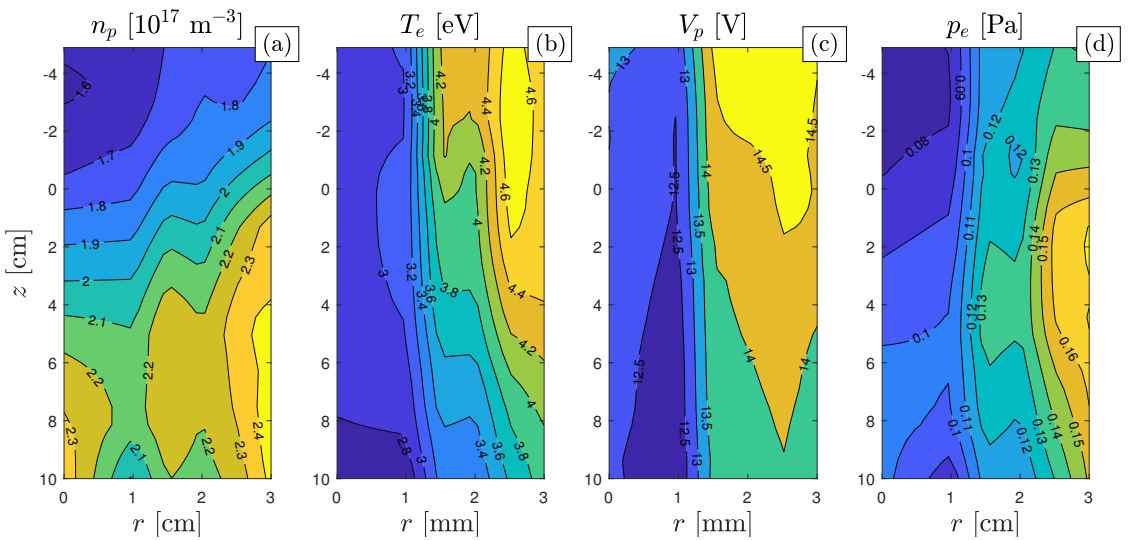

FIG. 19. Contour plot of (a) plasma density, (b) electron temperature, (c) plasma potential, (d) electron pressure. Input parameters are: $P_{I N}=750 \mathrm{~W}, \dot{m}=0.2 \mathrm{mg} / \mathrm{s} \mathrm{Xe}, 10 \mathrm{mT}$ peak value of the external B field.

Measurements of plasma properties are performed both axially and radially. On-axis scans of $n_{p}, T_{e}$ and $V_{p}$ are reported in Fig. 21(a)-(c) and Fig. 22(a)-(c) using krypton and xenon, respectively. Notably, substantial differences are drawn.

In Fig. 21(c), it is shown that the plasma potential changes from a rapidly dropping profile to an almost constant profile as the strength of the external magnetic field is increased. Furthermore, with reference to Fig. 21(a), the plasma density peak moves downstream. Visual inspection of the plasma source in operation agrees with these results. Indeed, at 4 and $5 \mathrm{mT}$, it is observed that plasma concentrates just below the antenna region, with almost null light emission elsewhere. On the contrary, a visibly homogeneous plasma column appears at $10 \mathrm{mT}$, extending over several antenna lengths. This strongly suggests that a magnetic field between 7 and $10 \mathrm{mT}$ is somewhat a threshold external parameter characterizing the transition from inductively-coupled regime to a wave-mode regime. Differently, when the plasma source operates with xenon, no mode transitions have been recorded. Qualitatively speaking, this is clearly shown in Fig. 22(a),(c) where the density and potential profiles shift up as the external magnetic field strength is increased. It has been previously reported the presence of density peaks at low externally applied magnetic fields ${ }^{44}$. In this particular experimental setup, either using krypton or xenon as working gas, no larger plasma density at lower magnetic fields has been recorded. Note that the density in Fig. 21 is larger than that in Fig. 22 because the reactor operates with a larger $\dot{m}$ for krypton, see Section IV. 

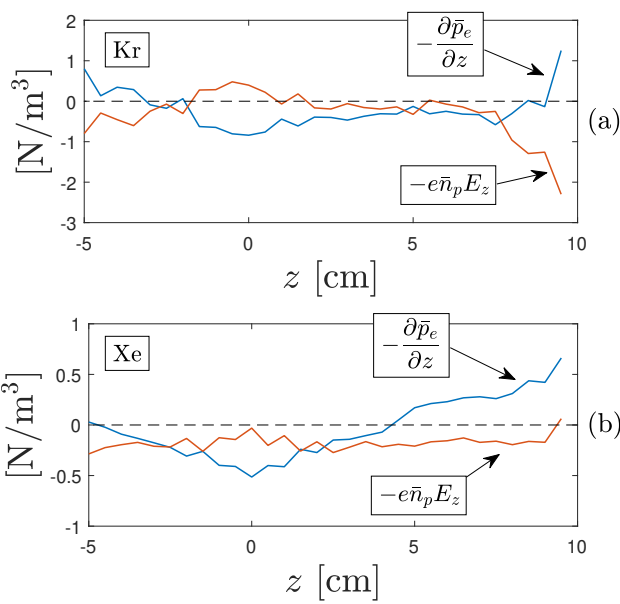

FIG. 20. Volumetric forces acting on the electrons for (a) krypton and (b) xenon propellants. Variables $\bar{p}_{e}$ and $\bar{n}_{p}$ refer to radially averaged quantities.

Detailed plasma properties measurements were also performed in the radial direction at $z=3 \mathrm{~cm}$ for the four different magnetic profiles. In accordance with the reference system in Fig. 1, the measurements were performed along the $x$ axis The results are given in Fig. 23(a)-(d) and Fig. 24(a)-(d) for krypton and xenon respectively. Filled dots represent measurement points and dotted lines are smoothed profiles to enhance data visualization. In agreement with Fig. 18 and 19, density, temperature and potential peak values are localized off-axis. Similar outcomes have been previously reported for $n_{p}$ and $T_{e}{ }^{45,46}$, but not for the plasma potential to the knowledge of the authors. It has been suggested that off-axis density peak is due to transport of high temperature electrons from the plasma discharge along the surface of the $\mathrm{MN}^{47}$. Fig. 23(a) and Fig. 24(a) show how ionization efficiency is enhanced at stronger magnetic fields. No relevant advantages in terms of electron temperature are obtained by increasing the applied field for krypton, as seen in Fig. 23(b). Differently, peak value of $T_{e}$ increases by more than $20 \%$ ( $4 \mathrm{mT}$ vs. $10 \mathrm{mT}$ ) when xenon is used, cf. Fig. 24(b). Similarly, $V_{p}$ profiles in Fig. 23(c) and Fig. 24(c) show that a more energetic plasma is obtained at larger B fields.

Neglecting the ion contribution to the plasma pressure $\left(T_{i} \ll T_{e}\right)$, the local $\beta$ parameter, i.e. particle pressure over magnetic pressure ${ }^{48}$, is computed using the pressure profiles illustrated in Fig. 23(d) and Fig. 24(d). Calculations yield $10^{-4} \lesssim \beta \lesssim 10^{-2}$, outlining a negligible diamagnetic effect in the proximity of the magnetic throat.

Radially non-uniform pressure and potential profiles shown in Fig. 23 and 24 inherently imply the presence of a Hall current $j_{\theta_{e}}$ in the expanding plasma, which has been analyzed theoretically/numerically ${ }^{24}$ and experimentally ${ }^{46}$. When de-
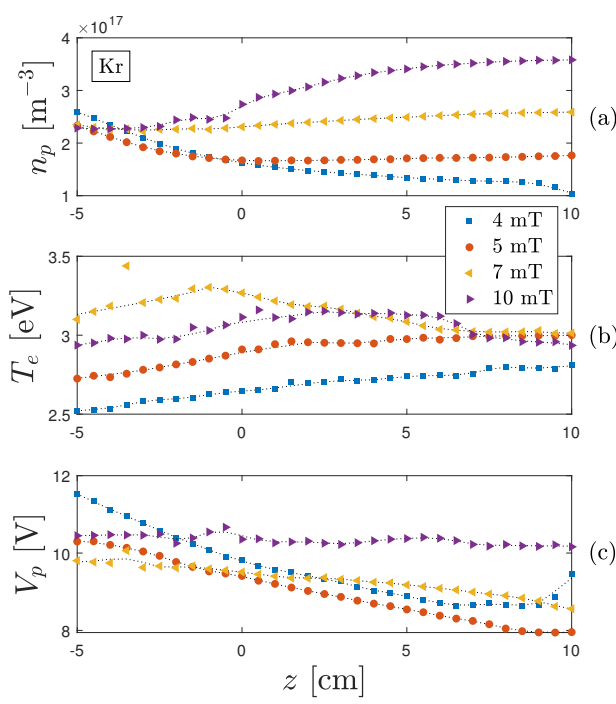

FIG. 21. Evolution along the reactor axis of (a) plasma density, (b) electron temperature and (c) plasma potential for different magnetic strengths. $P_{I N}=750 \mathrm{~W}, \dot{m}=1 \mathrm{mg} / \mathrm{s} \mathrm{Kr}, r=0 \mathrm{~mm}$.
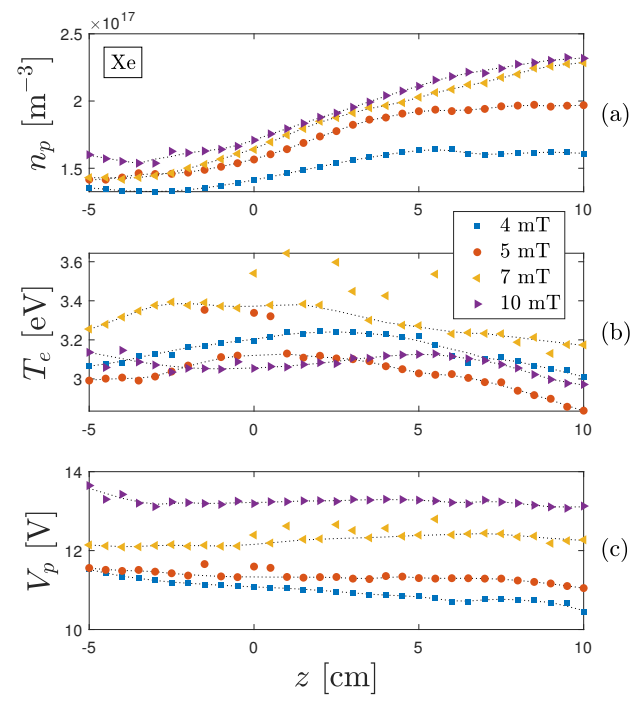

FIG. 22. Evolution along the reactor axis of (a) plasma density, (b) electron temperature and (c) plasma potential for different magnetic strengths. $P_{I N}=750 \mathrm{~W}, \dot{m}=0.2 \mathrm{mg} / \mathrm{s} \mathrm{Xe}, r=0 \mathrm{~mm}$. 

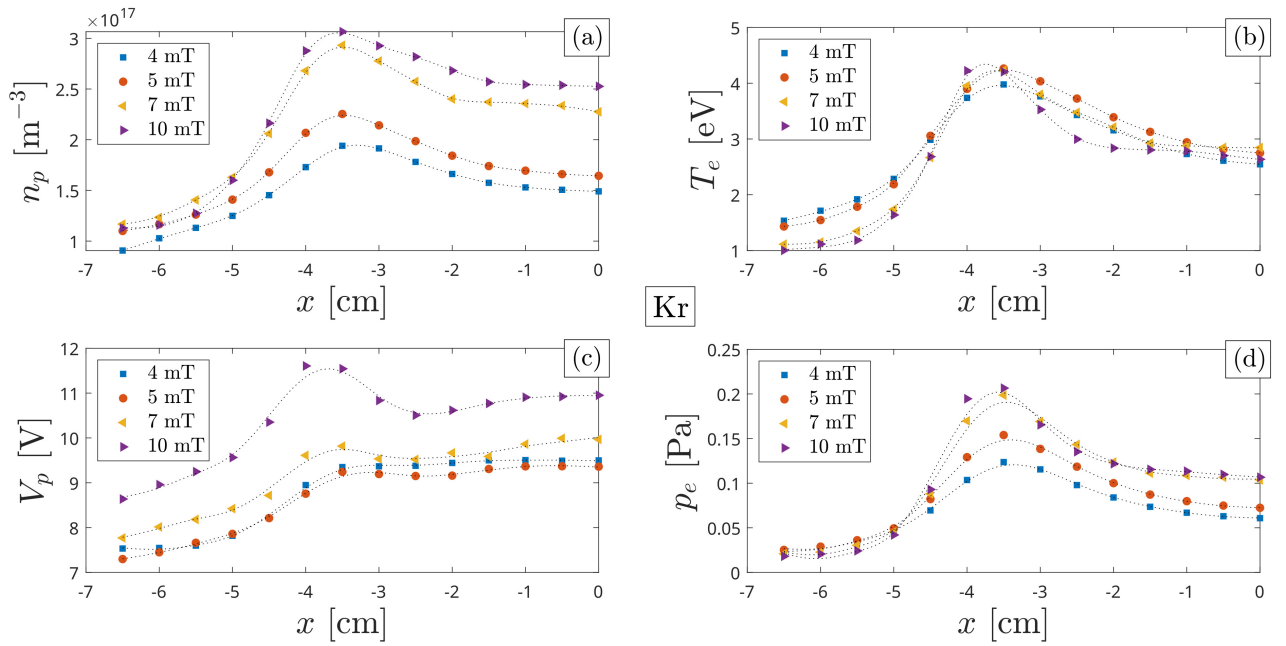

FIG. 23. Radial profile of (a) plasma density, (b) electron temperature, (c) plasma potential and (d) electron pressure for different magnetic strengths. $P_{I N}=750 \mathrm{~W}, \dot{m}=1 \mathrm{mg} / \mathrm{s} \mathrm{Kr}, z=3 \mathrm{~cm}$.
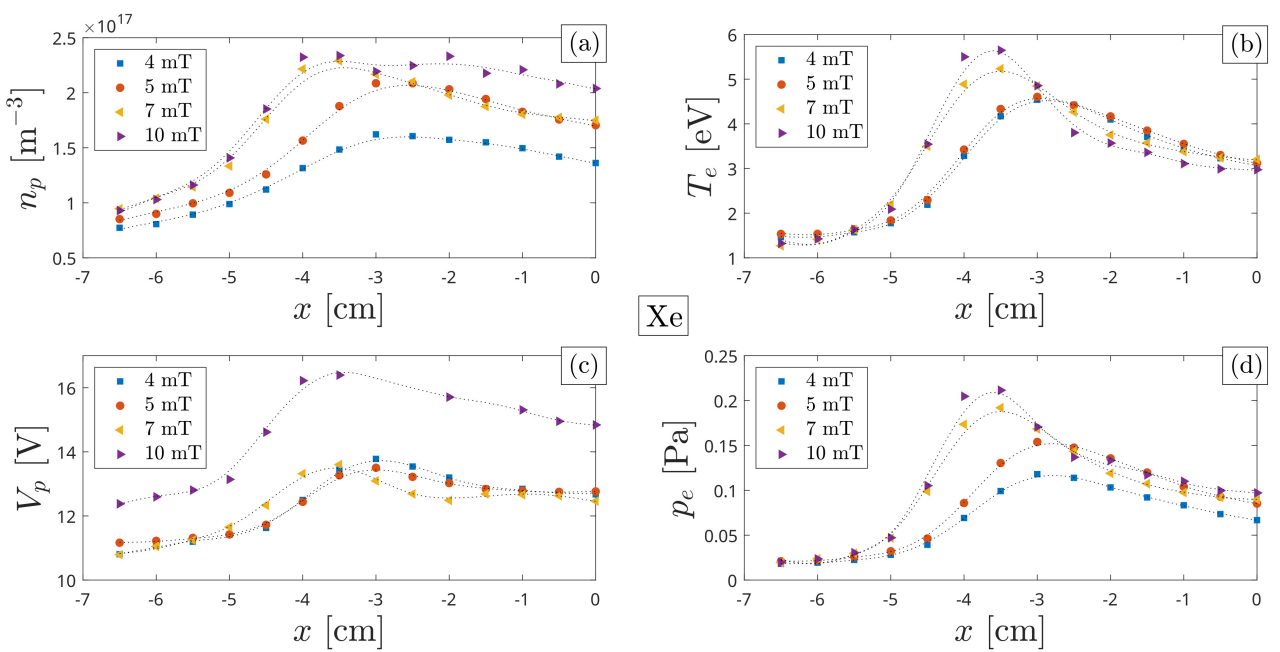

FIG. 24. Radial profile of (a) plasma density, (b) electron temperature, (c) plasma potential and (d) electron pressure for different magnetic strengths. $P_{I N}=750 \mathrm{~W}, \dot{m}=0.2 \mathrm{mg} / \mathrm{s} \mathrm{Xe}, z=3 \mathrm{~cm}$.

scribing the evolution of axial momentum in a single conducting fluid description, i.e. combining electrons and ions momentum equations, it is deduced that thrust generation mechanisms in a HP thruster are due to an electrothermal acceleration and an electromagnetic Hall acceleration ${ }^{24,49}$. The first contribution results from the plasma pressure acting inside the discharge chamber and on the "walls" of the diverging $\mathrm{MN}$, while the second one results from a Lorentz force proportional to the net azimuthal current $j_{\theta}$. However, it has been experimentally observed ${ }^{26}$ that the swirl current $j_{\theta_{i}}$, i.e. the ion contribution to $j_{\theta}$, is fairly negligible for the values of applied magnetic field in analysis as the ions are unmagnetized. The 

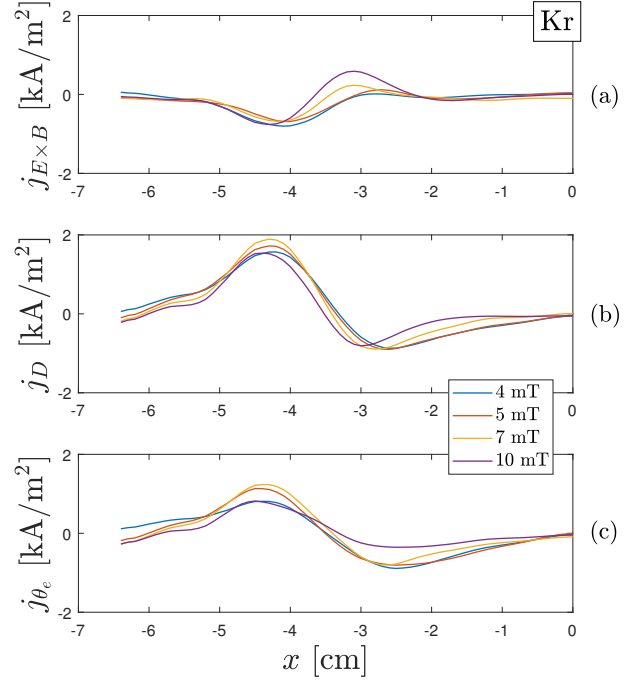

FIG. 25. Computed profiles of (a) electron $E \times B$ current density, (b) electron diamagnetic current density and (c) Hall current $j_{E \times B}+j_{D}$ from plasma properties in Fig. 23.
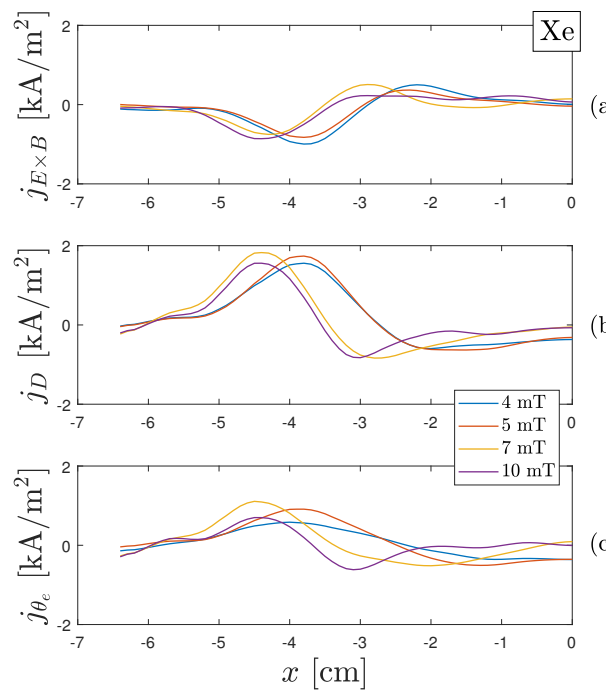

FIG. 26. Computed profiles of (a) electron $\mathrm{E} \times \mathrm{B}$ current density, (b) electron diamagnetic current density and (c) Hall current $j_{E \times B}+j_{D}$ from plasma properties in Fig. 24. profile of $j_{\theta_{e}}$ in this particular experiment can be inferred by using the radial component of the electron momentum equation in cylindrical coordinates, which reads

$$
-n_{p} e E_{r}+j_{\theta_{e}} B_{z}-\frac{\partial p_{e}}{\partial r}=0,
$$

where $E_{r}$ is the radial electric field, $B_{z}$ is the axial magnetic strength and the rest has already been introduced. It is shown that the Hall current comprises an $E \times B$ drift component $j_{E \times B}$ and a diamagnetic drift $j_{D}$ component:

$$
j_{\theta_{e}}=j_{E \times B}+j_{D}=n_{p} e \frac{E_{r}}{B_{z}}+\frac{1}{B_{z}} \frac{\partial p_{e}}{\partial r} .
$$

The smoothed profiles of $p_{e}$ and $V_{p}$ in Fig. 23 and Fig. 24 have been used to calculate the current densities reported in Fig. 25 and Fig. 26, for krypton and xenon respectively. Taylor Series Method (TSM) is used to evaluate the uncertainty associated with the indirect estimation of $j_{\theta_{e}}$ through Equation 4. It has been assumed that propagation of the error is only due to $n_{p}$, $T_{e}$ and $V_{p}$, whose error values are shown in Table I. By expanding Equation 4 and substituting partial derivatives with finite differentiation terms, the expression for the uncertainty of $j_{\theta_{e}}$ truncated to the first order reads ${ }^{50}$

$$
\begin{aligned}
\left(\frac{U_{j_{\theta_{e}}}}{j_{\theta_{e}}}\right)^{2}= & 2\left(\frac{U_{n_{p}}}{n_{p}}\right)^{2}+\left(\frac{U_{T_{e}}}{T_{e}}\right)^{2}+\left(\sqrt{2} \frac{U_{n_{p}}}{\Delta n_{p}}\right)^{2}+ \\
& +\left(\sqrt{2} \frac{U_{T_{e}}}{\Delta T_{e}}\right)^{2}+\left(\sqrt{2} \frac{U_{V_{p}}}{\Delta V_{p}}\right)^{2} .
\end{aligned}
$$

Typical values of $U_{j_{\theta_{e}}} / j_{\theta_{e}}$ are in the order of $3 \%$ for all operating conditions in terms of magnetic field strength and propellant. Note that $j_{\theta_{e}}>0$ at $x<0$ indicates that current flows in the diamagnetic direction (i.e. the magnetic field associated to the net $j_{\theta}$ is directed along $-z$ ), while $j_{\theta_{e}}<0$ at $x<0$ indicates it flows in the anti-diamagnetic direction. No profound differences are found when using krypton or xenon. With both propellants, $j_{E \times B}$ flows in the diamagnetic direction in the inner radial region and in the anti-diamagnetic direction in the outer radial region, cf. Fig. 25(a) and Fig. 26(a). The opposite behavior is recorded for $j_{D}$, cf. Fig. 25(b) and Fig. 26(b). Since the local thrust component due to the Lorentz force expressed in cylindrical coordinates is proportional to the product $r j_{\theta_{e}} B_{r}$, only values of $j_{\theta_{e}}$ flowing in the positive $\theta$ direction contribute to thrust generation. In the Cartesian reference system presented here, positive thrust contribution is given by $j_{\theta_{e}}>0$ at $x<0$ and vice versa. At the specific axial location

$$
\begin{array}{c|c|c|c|c|c}
\multicolumn{5}{c}{\int_{0}^{\left|x_{\max }\right|}|x| j_{\theta_{e}} \mathrm{~d} x} & {[\mathrm{~A}]} \\
& 4 \mathrm{mT} & 5 \mathrm{mT} & 7 \mathrm{mT} & 10 \mathrm{mT} \\
\hline \hline \mathrm{Kr} & 0.26 & 0.27 & 0.35 & 0.25 \\
\mathrm{Xe} & 0.34 & 0.49 & 0.40 & 0.15 \\
\hline
\end{array}
$$

TABLE II. Mean cumulative values of $|x| j_{\theta_{e}}$ integration along $|x|$ for krypton and xenon propellants at different magnetic strengths. 


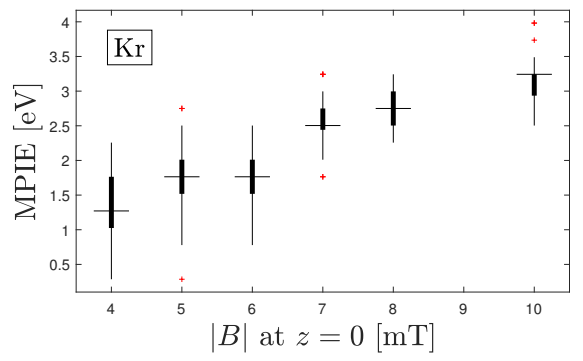

FIG. 27. Most probable ion energy as function of the magnetic field. $P_{I N}=750 \mathrm{~W}, \dot{m}=1 \mathrm{mg} / \mathrm{s} \mathrm{Kr}, z=33 \mathrm{~cm}$. Horizontal mark indicates median value, thick box delimits 25 th and 75 th percentiles, red marks represent outliers.

where the radial scans are performed, integration over the radial direction outlines that a net thrust is produced for all the magnetic field magnitudes. Numerical values are reported in Table II. Accounting for $B_{r}$ in the integral does not contribute in sign changing as it is positive in the $r$ direction. Estimation of the total thrust would require a complete map of the plume and integration over the plasma volume.

Additional measurements include the investigation of ion energy as function of the externally applied magnetic field In doing so, the RPA detailed in Section IIID has been employed. To increase data resolution, magnetic profiles with $6 \mathrm{mT}$ and $8 \mathrm{mT}$ peak values have been added to the four ones previously discussed. The probe has been placed downstream at $z=33 \mathrm{~cm}$. While operating the HP source at one specific magnetic configuration, fifty $\mathrm{I}-\mathrm{V}$ characteristics are acquired in groups of ten. Data is firstly smoothed and later numerically derived to obtain the ion energy distribution function (IEDF).

The most probable ion energy (MPIE) is then extracted as the IEDF peak value. Results in terms of MPIEs for krypton and xenon are reported in Fig. 27 and Fig. 28, respectively. As expected, higher ion energies are measured as the external magnetic field strength is increased, coherently with what reported in Fig. 21(c) and Fig. 22(c). Generally speaking, although consistent with the values of plasma potential, typical ion energies herein reported are very small, suggesting low propulsive performance of the HP source in analysis. The measured ion energies imply electrostatic ion speeds of about $2.7 \mathrm{~km} / \mathrm{s}$ for $\mathrm{Kr}^{+}$and about $3.5 \mathrm{~km} / \mathrm{s}$ for $\mathrm{Xe}^{+}$, both estimated for the highest strength of the magnetic field. Higher beam energies in the range of few tens of $\mathrm{eV}$ have been reported in previous works ${ }^{7,29,51-53}$.It is reasonable to state that this large performance discrepancy with respect to other HP sources comes from a complete different set of input parameters. The source analyzed in this work features a relatively large geometry and operated at relatively weak magnetic fields and small mass flow rates.

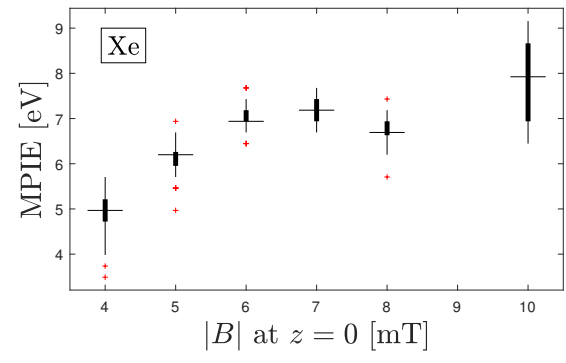

FIG. 28. Most probable ion energy as function of the magnetic field $P_{I N}=750 \mathrm{~W}, \dot{m}=0.2 \mathrm{mg} / \mathrm{s} \mathrm{Xe}, z=33 \mathrm{~cm}$. Horizontal mark indicates median value, thick box delimits 25 th and 75 th percentiles, red marks represent outliers.

\section{CONCLUSION}

Several plasma properties have been inferred via direct measurement in a HP source operating with krypton and xenon as propellants. The magnetic component of the rf field in the plasma is axially characterized with and without the external magnetic field, showing that an electromagnetic wave propagates downstream in the first case only. Plasma density, electron temperature and plasma potential have been determined both axially and radially for different configurations of the externally applied magnetic field. 2-D profiles of these properties are then provided when the HP source operates with a magnetic field peak value of $10 \mathrm{mT}$ at the exit cross section of the discharge chamber. It is found that the electron temperature peaks off-axis just before the exit cross section, while the peak in plasma density occurs similarly off-axis but downstream where the electrons are cooler. This phenomenon is quantitatively attributed to pressure balance effects. Using the plasma density data for the two propellants and their related mass flow rates, it is computed that xenon is roughly 5 times more efficiently ionized in the probed region. Qualitatively and quantitatively different plasma potential axial profiles are reported when using krypton for distinct external magnetic fields strengths, thus suggesting that the plasma source operates in inductive mode below a threshold value of the applied field. On the contrary, no mode transitions have been found when using xenon, which operates in helicon mode even for the weakest magnetic field. By using radial measurements performed few centimeters downstream the tube exit, the Hall current is computed as the sum of a $E \times B$ contribution and a diamagnetic contribution. Radial integration of the Hall current shows that a positive net thrust is locally produced. Ion energy is measured as function of the applied magnetic field strengths, showing that xenon performs better. Nevertheless, low ion energies are recorded.

The present work provides an useful dataset to be exploited in developing theoretical models and numerical simulations of the plasma expansion in the MN. A complete 2-D description of krypton and xenon plasma plume would enable the eval- 
uation of the attained performance thus guiding the thruster design procedure. Investigation of smaller size devices and different topologies of the magnetic field are certainly of interest for performance improvement and thruster optimization. Examination of the multiply-charged species fraction in the plume would further refine the knowledge of such plasma sources.

\section{ACKNOWLEDGMENTS}

This project has received funding from the European Union's Horizon 2020 research and innovation program under grant agreement No 870542 (HelIcon PlasmA Thruster for In-space Applications).

\section{DATA AVAILABILITY}

The data that support the findings of this study are available from the corresponding author upon reasonable request.

${ }^{1}$ R. W. Boswell, "Very efficient plasma generation by whistler waves near the lower hybrid frequency," Plasma Physics and Controlled Fusion 26, 1147 1162 (1984).

${ }^{2}$ F. F. Chen, "Plasma ionization by helicon waves," Plasma Physics and Controlled Fusion 33, 339-364 (1991).

${ }^{3}$ F. F. Chen, M. J. Hsieh, and M. Ligh, "Helicon waves in a non-uniform plasma," Plasma Sources Sci. Technol. 3, 49-57 (1993).

${ }^{4}$ F. F. Chen, "Physics of helicon discharges," Physics of Plasmas 3, 1783 1793 (1996).

${ }^{5}$ I. D. Sudit and F. F. Chen, "Discharge equilibrium of a helicon plasma," Plasma Sources Science and Technology 5, 43-53 (1996).

${ }^{6}$ A. W. Degeling, C. O. Jung, R. W. Boswell, and A. R. Ellingboe, "Plasm production from helicon waves," Physics of Plasmas 3, 2788-2796 (1996) ${ }^{7}$ O. V. Batishchev, "Minihelicon plasma thruster," IEEE Transactions on Plasma Science 37, 1563-1571 (2009).

${ }^{8} \mathrm{E}$. Ahedo and M. Merino, "On plasma detachment in propulsive magnetic nozzles," Physics of Plasmas 18, 1-8 (2011).

${ }^{9}$ K. Takahashi, T. Lafleur, C. Charles, P. Alexander, R. W. Boswell, M. Perren, R. Laine, S. Pottinger, V. Lappas, T. Harle, and D. Lamprou, "Direct thrust measurement of a permanent magnet helicon double layer thruster," Applied Physics Letters 98, 2-4 (2011).

${ }^{10} \mathrm{M}$. Merino and E. Ahedo, "Plasma detachment in a propulsive magnetic nozzle via ion demagnetization," Plasma Sources Science and Technology 23 (2014), 10.1088/0963-0252/23/3/032001.

${ }^{11}$ A. Cardinali, D. Melazzi, M. Manente, and D. Pavarin, "Ray-tracin WKB analysis of Whistler waves in non-uniform magnetic fields applied to space thrusters," Plasma Sources Science and Technology 23 (2014), $10.1088 / 0963-0252 / 23 / 1 / 015013$

${ }^{12}$ M. Magarotto, D. Melazzi, and D. Pavarin, "Study on the influence of the magnetic field geometry on the power deposition in a helicon plasma source," Journal of Plasma Physics 85, 1-19 (2019).

${ }^{13}$ M. Magarotto and D. Pavarin, "Parametric Study of a Cathode-Less Radio Frequency Thruster," IEEE Transactions on Plasma Science, 1-13 (2020). ${ }^{4}$ M. Magarotto, M. Manente, F. Trezzolani, and D. Pavarin, "Numerica Model of a Helicon Plasma Thruster," IEEE Transactions on Plasma Science , $1-10(2020)$

${ }^{15}$ M. Magarotto, D. Melazzi, and D. Pavarin, "3D-VIRTUS: Equilibrium condition solver of radio-frequency magnetized plasma discharge for space applications," Computer Physics Communications 247, 106953 (2020)

${ }^{16}$ K. Takahashi, Y. Takao, and A. Ando, "Increased Thrust-to-Power Ratio of a Stepped-Diameter Helicon Plasma Thruster with Krypton Propellant," Journal of Propulsion and Power (2020), 10.2514/1.J054179.
${ }^{17}$ K. Takahashi, C. Charles, R. W. Boswell, Y. Takao, A. Fruchtman, J. Navarro-Cavalleé, and M. Merino, "Commentary: On helicon thrusters: Will they ever fly?" Frontiers in Physics (2020), 10.1063/1.5139998.

${ }^{18}$ E. Dale, B. Jorns, and A. Gallimore, "Future Directions for Electric Propulsion Research," Aerospace 7 (2020).

${ }^{19} \mathrm{M}$. Manente, F Trezzolani, M. Magarotto, D Pavarin, E. Fantino, M. Manente, F. Trezzolani, M. Magarotto, D. Pavarin, E. Fantino,
A. Selmo, N. Bellomo, and E. Toson, "REGULUS : A propulsion platA. Selmo, N. Bellomo, and E. Toson, "REGULUS : A propulsion plat-
form to boost small satellite missions," Acta Astronautica 157, 241-249 (2019).

${ }^{20}$ M. Ruiz, V. Gomez, P. Fajardo, J. Navarro-Cavalleé, R. Albertoni, G. Dickeli, A. Vinci, S. Mazouffre, and N. Hildebrand, "HIPATIA: A project for the development of the Helicon Plasma Thruster and its associated technologies to intermediate-high TRLs," in 71 st International Astronautical Congress (IAC) - The CyberSpace Edition (2020).

${ }^{21}$ M. Light, I. D. Sudit, F. F. Chen, and D. Arnush, "Axial propagation of helicon waves," Physics of Plasmas 2, 4094-4103 (1995).

${ }^{22}$ D. D. Blackwell, T. G. Madziwa, D. Arnush, and F. F. Chen, "Evidence for Trivelpiece-Gould Modes in a Helicon Discharge," Physical Review Letters 88, 4 (2002).

${ }^{23}$ S. A. Andersen, V. O. Jensen, P. Nielsen, and N. D'Angelo, "Continuous supersonic plasma wind tunnel," Physics of Fluids 12, 557-560 (1969).

${ }^{24} \mathrm{E}$. Ahedo and M. Merino, "Two-dimensional supersonic plasma acceleration in a magnetic nozzle," Physics of Plasmas 17, 1-15 (2010).

${ }^{25}$ J. M. Little and E. Y. Choueiri, "Critical condition for plasma confinement in the source of a magnetic nozzle flow," IEEE Transactions on Plasma Science 43, 277-286 (2015)

${ }^{26} \mathrm{~K}$. Takahashi, S. Takayama, A. Komuro, and A. Ando, "Standing Helicon Wave Induced by a Rapidly Bent Magnetic Field in Plasmas," Physical Review Letters 116, 1-5 (2016).

${ }^{27} \mathrm{~K}$. Takahashi and A. Ando, "Enhancement of axial momentum lost to the radial wall by the upstream magnetic field in a helicon source," Plasma Physics and Controlled Fusion 59 (2017), 10.1088/1361-6587/aa626f.

${ }^{28}$ R. M. Magee, M. E. Galante, J. Carr, G. Lusk, D. W. McCarren, and E. E. Scime, "Neutral depletion and the helicon density limit," Physics of Plasmas 20, 1-6 (2013).

${ }^{29} \mathrm{C}$. Charles and R. W. Boswell, "Effect of exhaust magnetic field in a helicon double-layer thruster operating in Xenon," IEEE Transactions on Plasma Science 36, 2141-2146 (2008)

${ }^{30} \mathrm{~F}$. Gesto, C. Charles, and R. Boswell, "Xenon ion beam detachment from a helicon double layer thruster," IEEE Transactions on Plasma Science 36, 1194-1195 (2008)

${ }^{31}$ J. Navarro-Cavallé, M. Wijnen, P. Fajardo, and E. Ahedo, "Experimental characterization of a $1 \mathrm{~kW}$ Helicon Plasma Thruster," Vacuum 149, 69-73 (2018)

${ }^{32}$ R. Jacquier, R. Agnello, B. Pouradier Duteil, P. Guittienne, A. Howling, G. Plyushchev, C. Marini, A. Simonin, I. Morgal, S. Bechu, and I. Furno, "First B-dot measurements in the RAID device, an alternative negative ion source for DEMO neutral beams," (2019).

${ }^{33} \mathrm{C}$. M. Franck, O. Grulke, and T. Klinger, "Magnetic fluctuation probe design and capacitive pickup rejection," Review of Scientific Instruments $\mathbf{7 3}$ 3768 (2002).

${ }^{34}$ M. P. Reilly, W. Lewis, and G. H. Miley, "Magnetic field probes for use in radio frequency plasma," Review of Scientific Instruments 80 (2009), 10.1063/1.3136907

${ }^{35}$ D. Gerst, M. Cirisan, S. Mazouffre, and A. Aanesland, "Investigation of Ion-Ion Plasmas for Application in electric thrusters," in IEPC (2011) pp. $1-7$.

${ }^{36}$ I. D. Sudit and F. F. Chen, "RF Compensated Probes for High-density Discharges," Plasma Sources Science and Technology 3, 162-168 (1994). ${ }^{37}$ F. F. Chen, "Lectures Notes on Langmuir Probe Diagnostics," (2003).

${ }^{38}$ F. F. Chen, "Langmuir probes in RF plasma: Surprising validity of OML theory," Plasma Sources Science and Technology 18 (2009), 10.1088/0963$0252 / 18 / 3 / 035012$

${ }^{39}$ I. H. Hutchinson, Principles of Plasma Diagnostics (2002).

${ }^{40}$ C. Niu, G. Zhao, Y. Wang, Z. Liu, and Q. Chen, "Correlation of wave propagation modes in helicon plasma with source tube lengths," Physics of Plasmas 24 (2017)

${ }^{41} \mathrm{P}$. Chabert and N. Braithwaite, Physics of Radio-Frequency Plasmas (Cambridge University Press, 2011) 
${ }^{42} \mathrm{D}$. Bose, T. R. Govidan, and M. Meyyappan, "Modeling of a helicon plasma source," IEEE TRANSACTIONS ON PLASMA SCIENCE, 3 (2003), 10.1109/PPPS.2001.01002007.

${ }^{43}$ B. v7.1 database, www. Ixcat. net, retrieved on November 8, 2020.

${ }^{44} \mathrm{~F}$. F. Chen, "Experiments on helicon plasma sources," Journal of Vacuum Science \& Technology A: Vacuum, Surfaces, and Films 10, 1389-1401 (1992).

${ }^{45} \mathrm{C}$. Charles, "High density conics in a magnetically expanding helicon plasma," Applied Physics Letters 96, 13-16 (2010)

${ }^{46}$ K. Takahashi, A. Chiba, A. Komuro, and A. Ando, "Experimental identification of an azimuthal current in a magnetic nozzle of a radiofrequency plasma thruster," Plasma Sources Science and Technology 25, 55011 (2016).

${ }^{47}$ K. Takahashi, C. Charles, R. Boswell, W. Cox, and R. Hatakeyama, "Transport of energetic electrons in a magnetically expanding helicon double laye plasma," Applied Physics Letters 94, 2007-2010 (2009).

${ }^{48}$ F. F. Chen, Introduction to Plasma Physics and Controlled Fusion (2016) ${ }^{49}$ K. Takahashi, T. Lafleur, C. Charles, P. Alexander, and R. W. Boswell, "Axial force imparted by a current-free magnetically expanding plasma," Physics of Plasmas 19, 1-9 (2012).

${ }^{50} \mathrm{H}$. W. Coleman and W. G. Steele, Experimentation, Validation, and Uncertainty Analysis for Engineers (Wiley, 2009).

${ }^{51}$ C. Charles and R. W. Boswell, "Laboratory evidence of supersonic ion beam generated by a current-free "helicon" double-layer," Physics of Plasmas 11, 1706-1714 (2004).

${ }^{52}$ T. Lafleur, C. Charles, and R. W. Boswell, "Characterization of the ion beam formed in a low magnetic field helicon mode," Journal of Physics D: Applied Physics 44 (2011), 10.1088/0022-3727/44/14/145204.

${ }^{53} \mathrm{~N}$. Gulbrandsen and $\AA$. Fredriksen, "RFEA measurements of high-energy electrons in a helicon plasma device with expanding magnetic field," Frontiers in Physics 5, 1-8 (2017). 


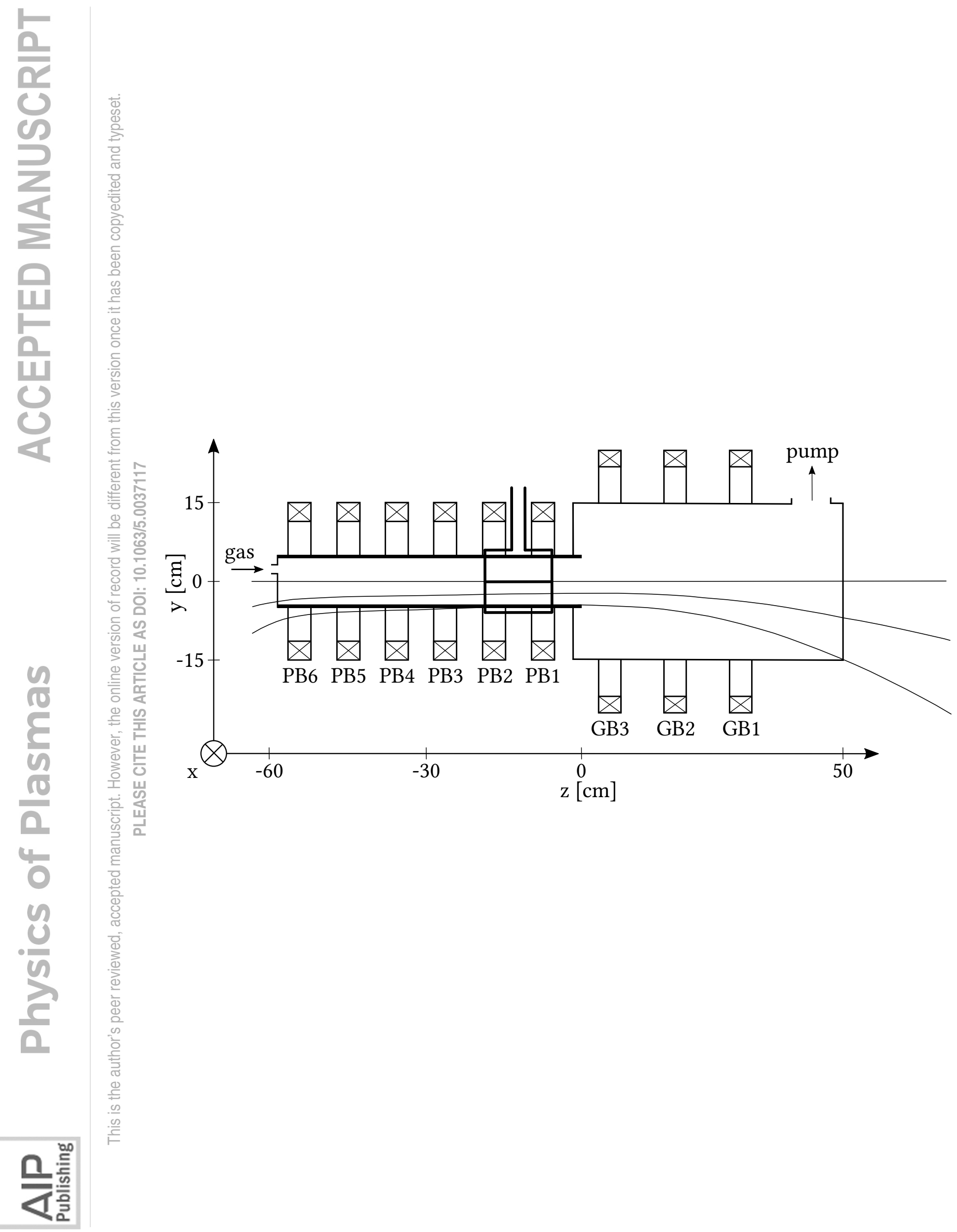




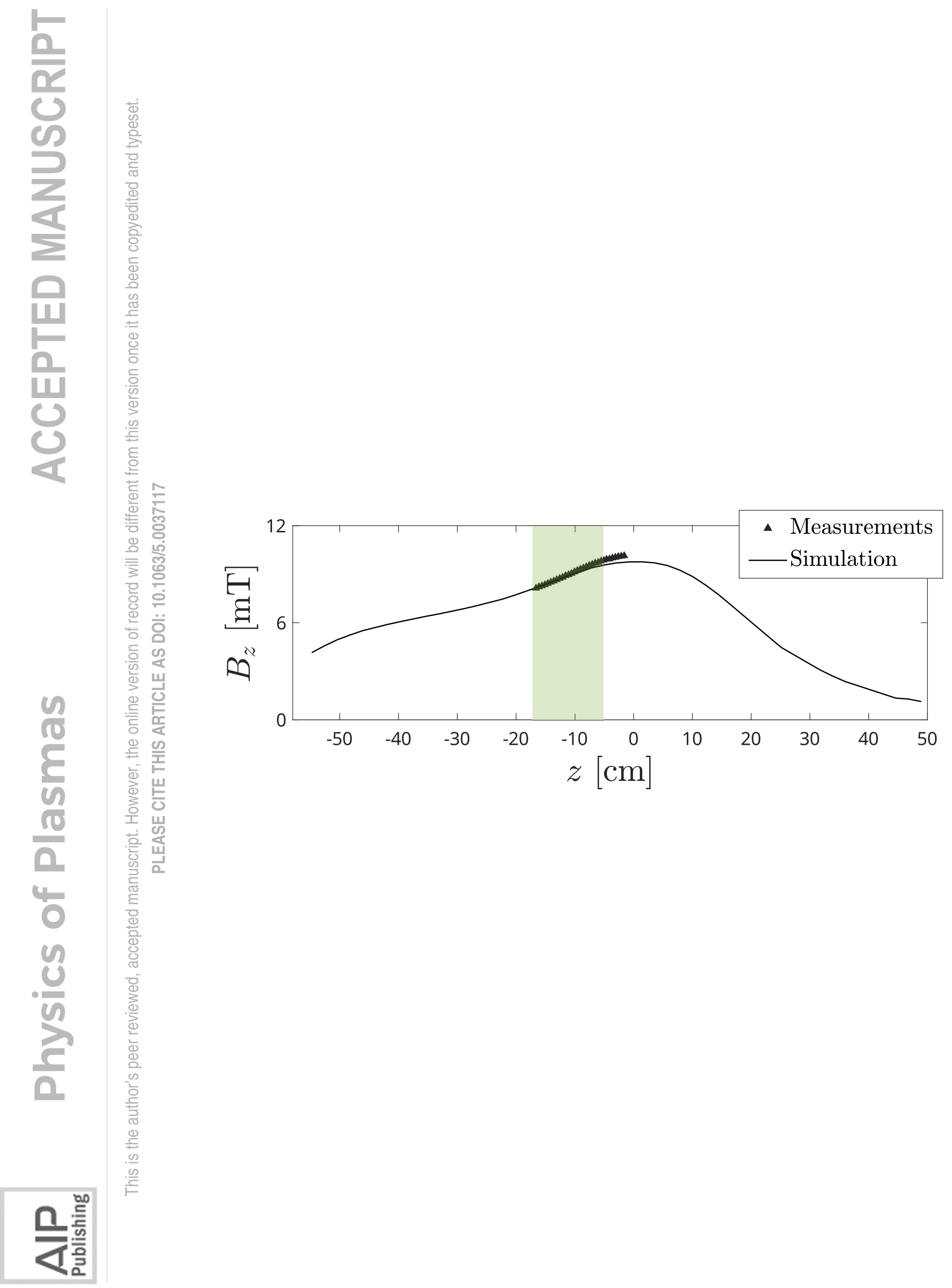




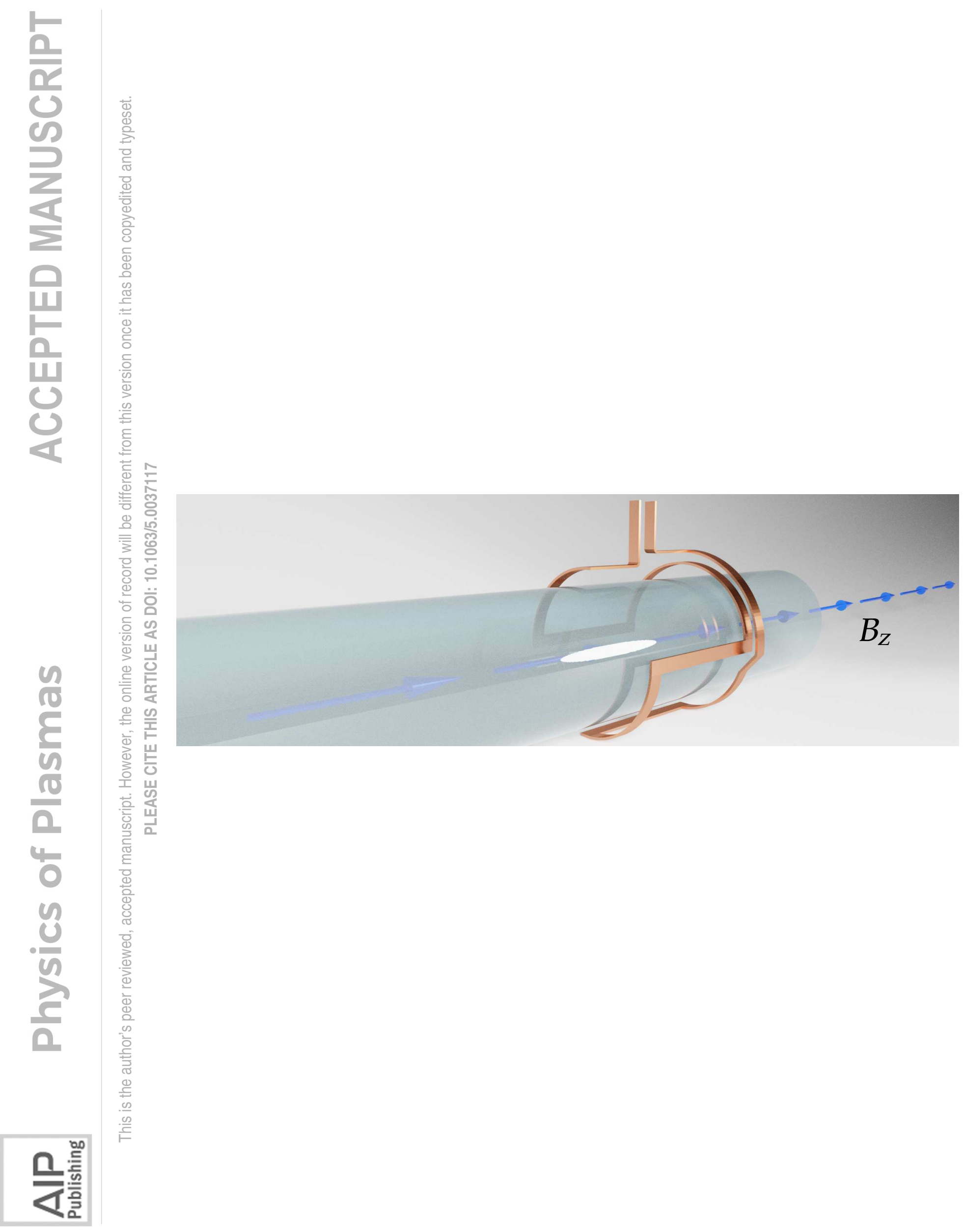




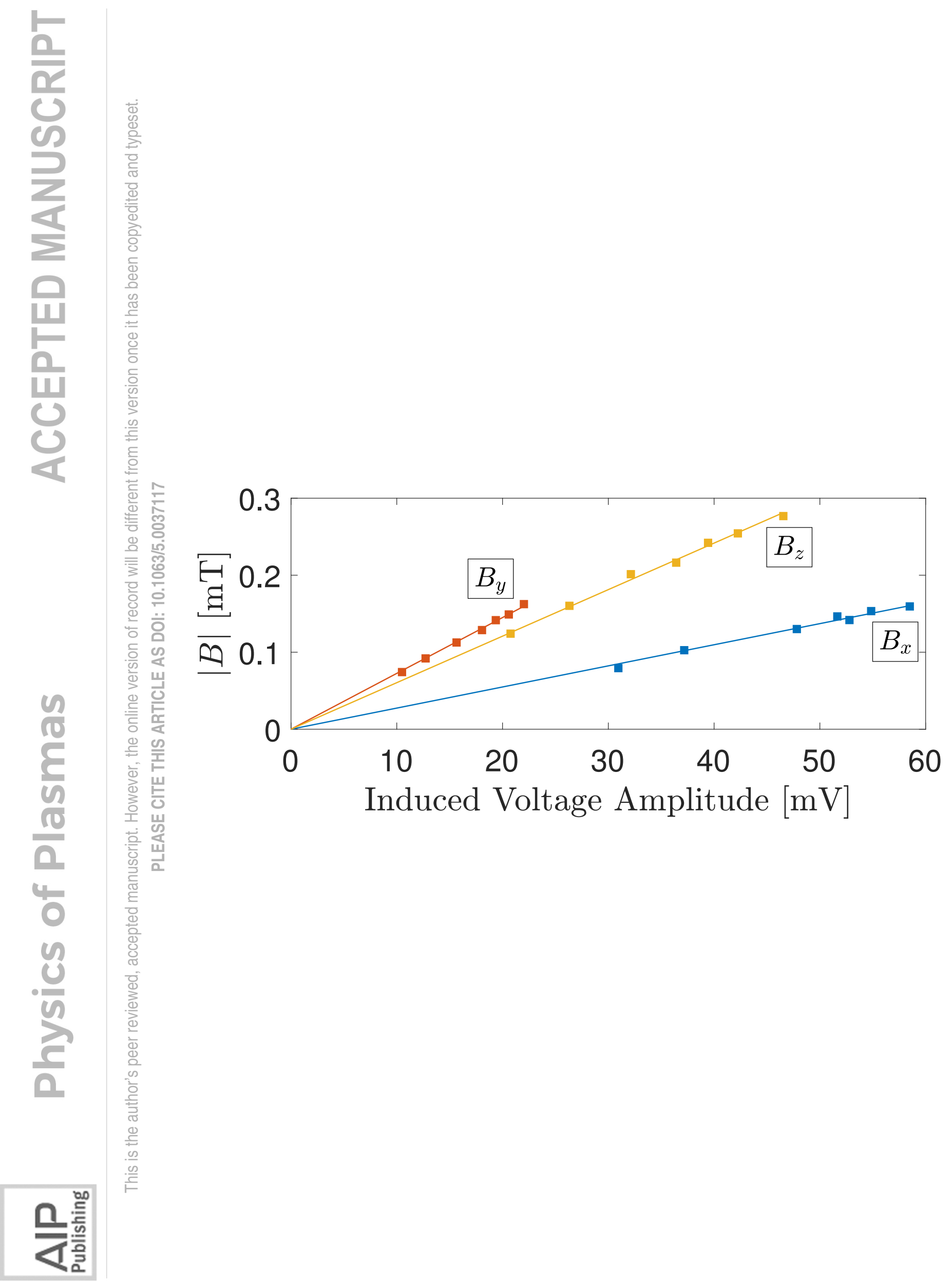




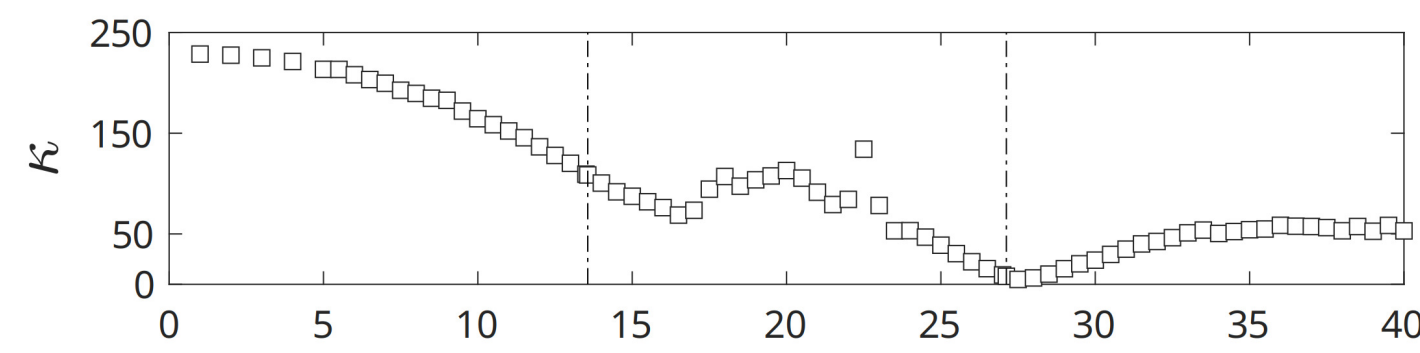

(a)

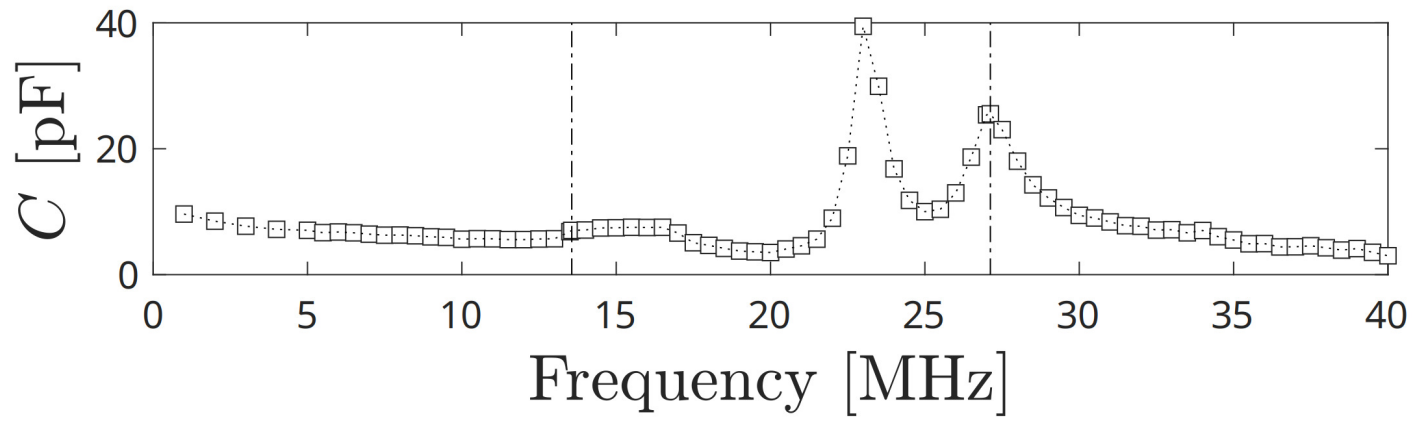

(b) 


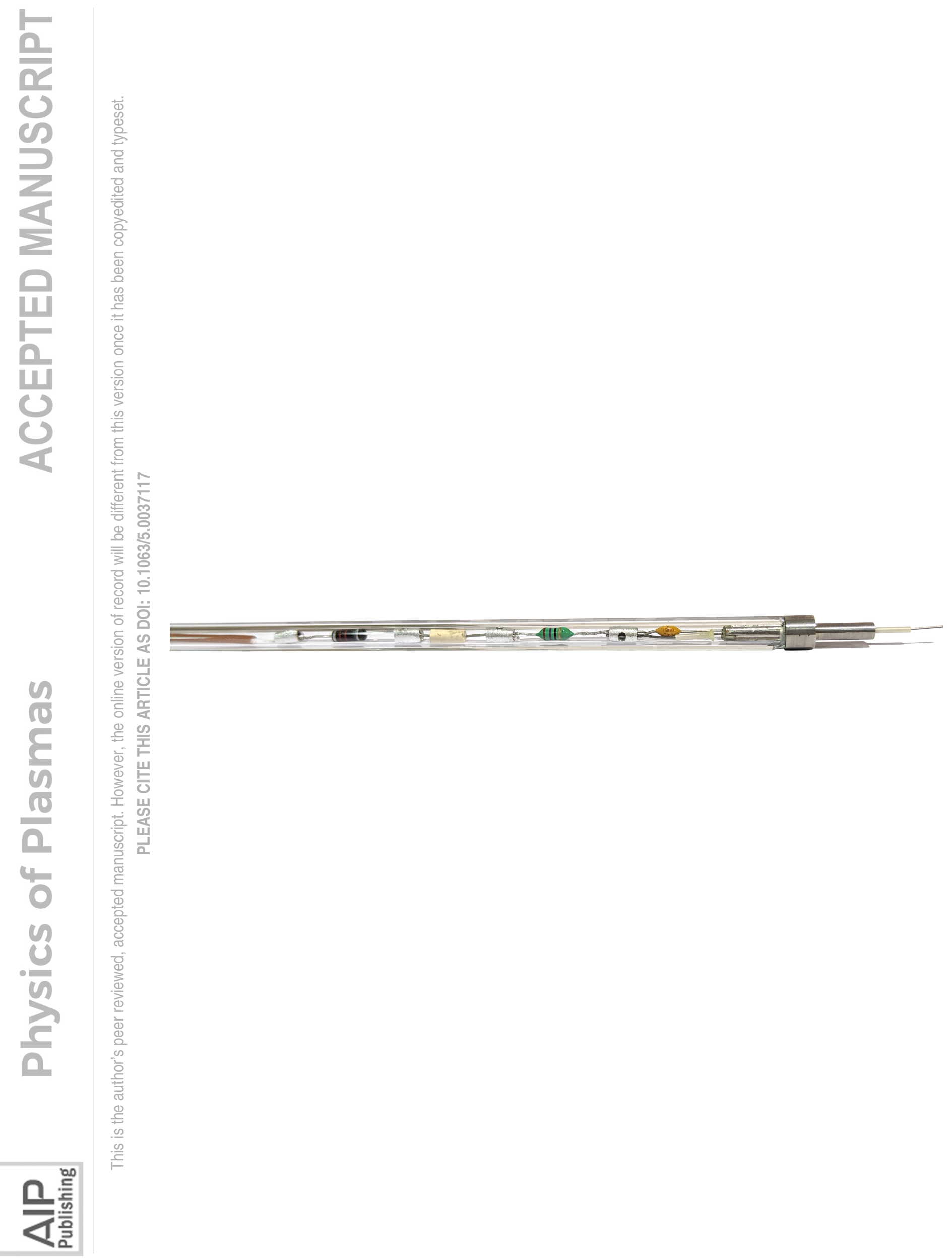




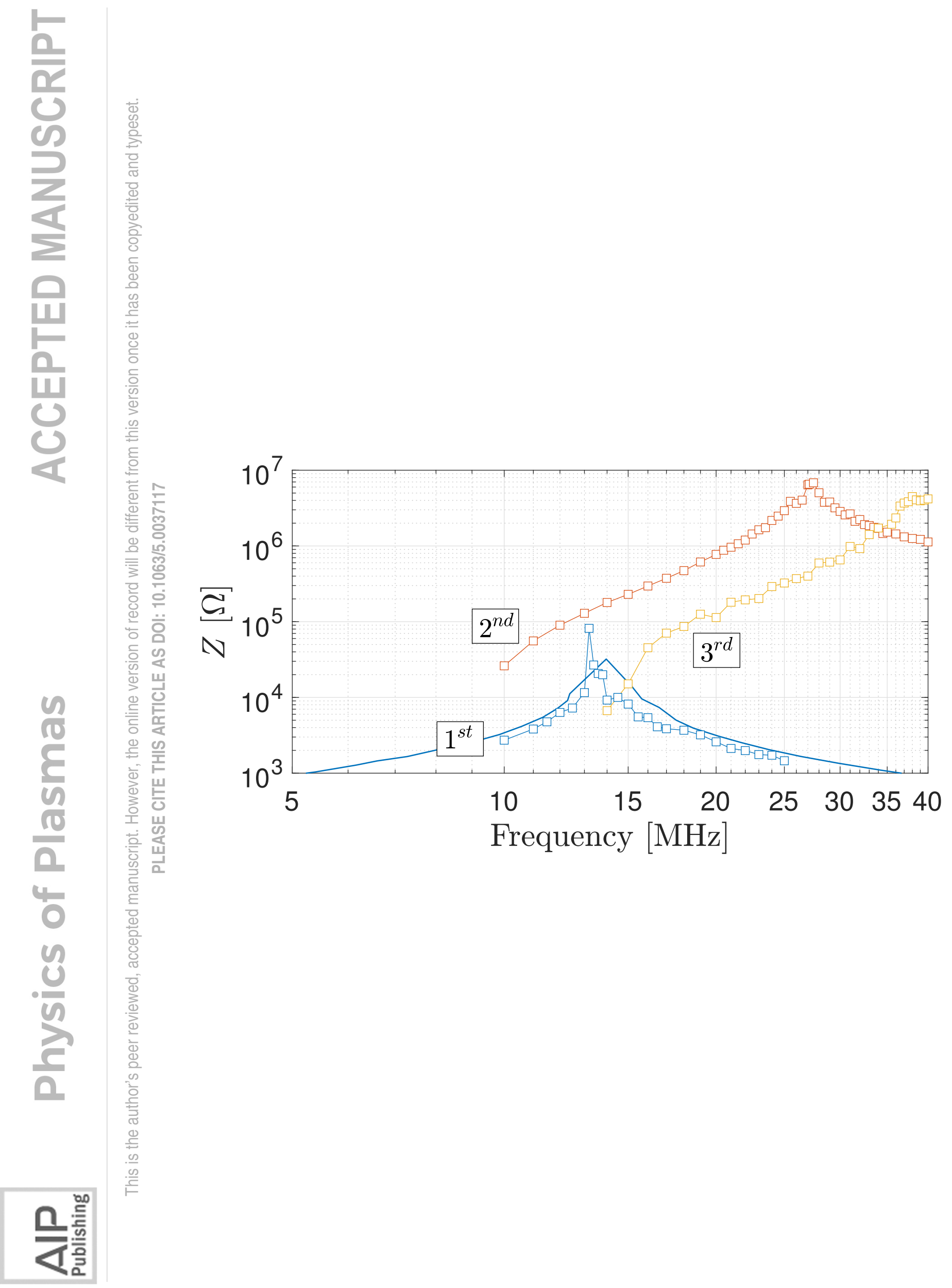




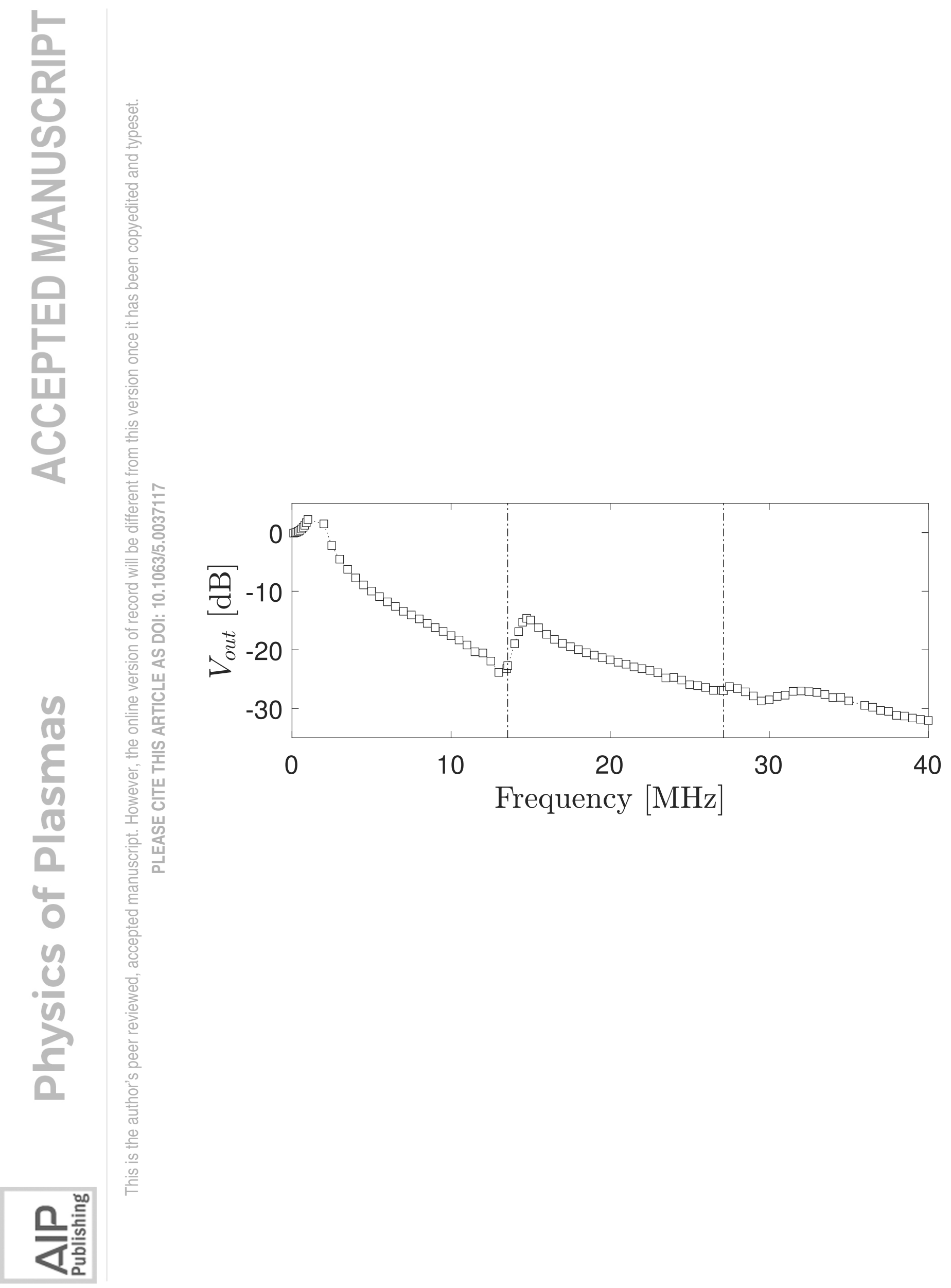




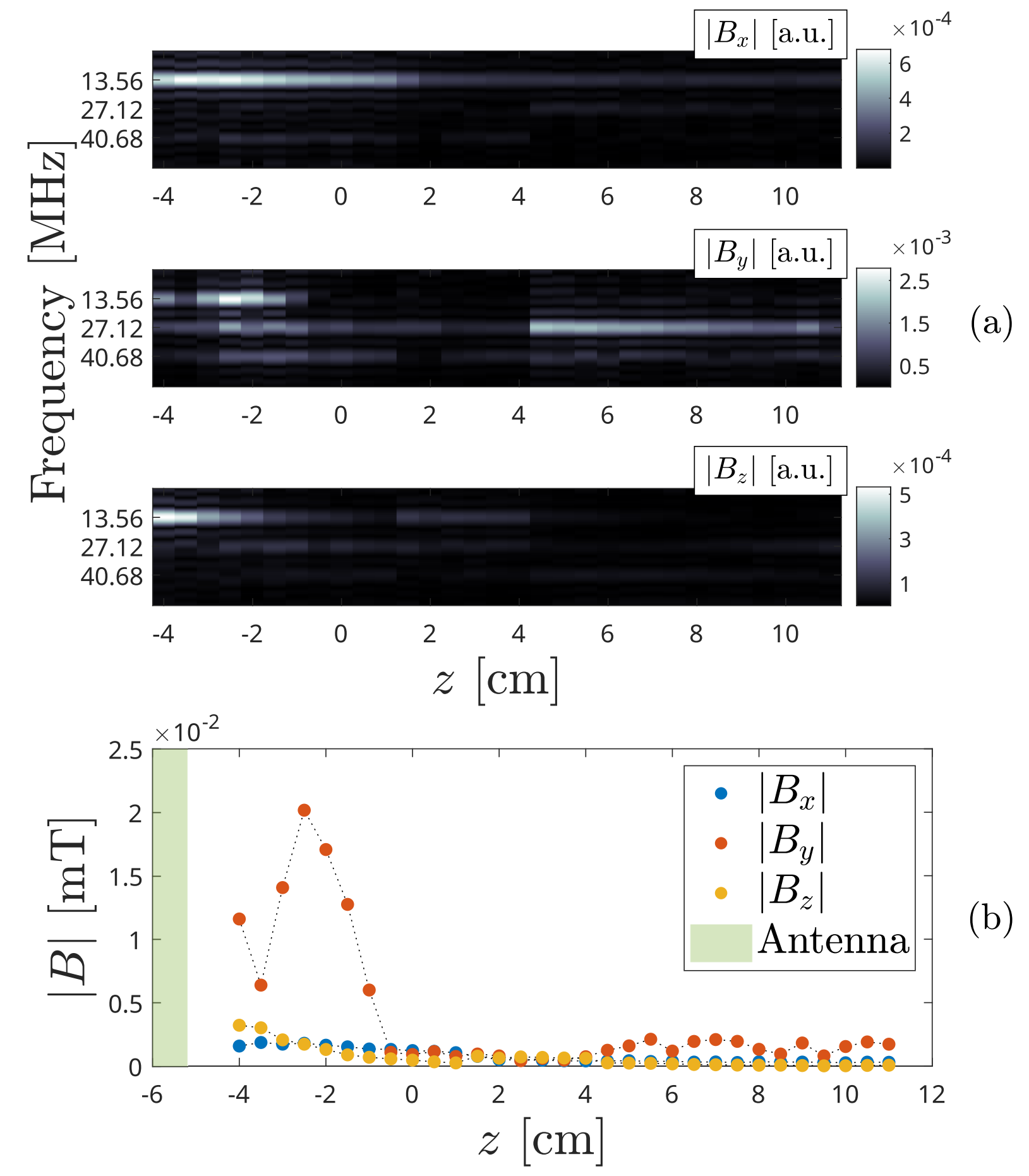




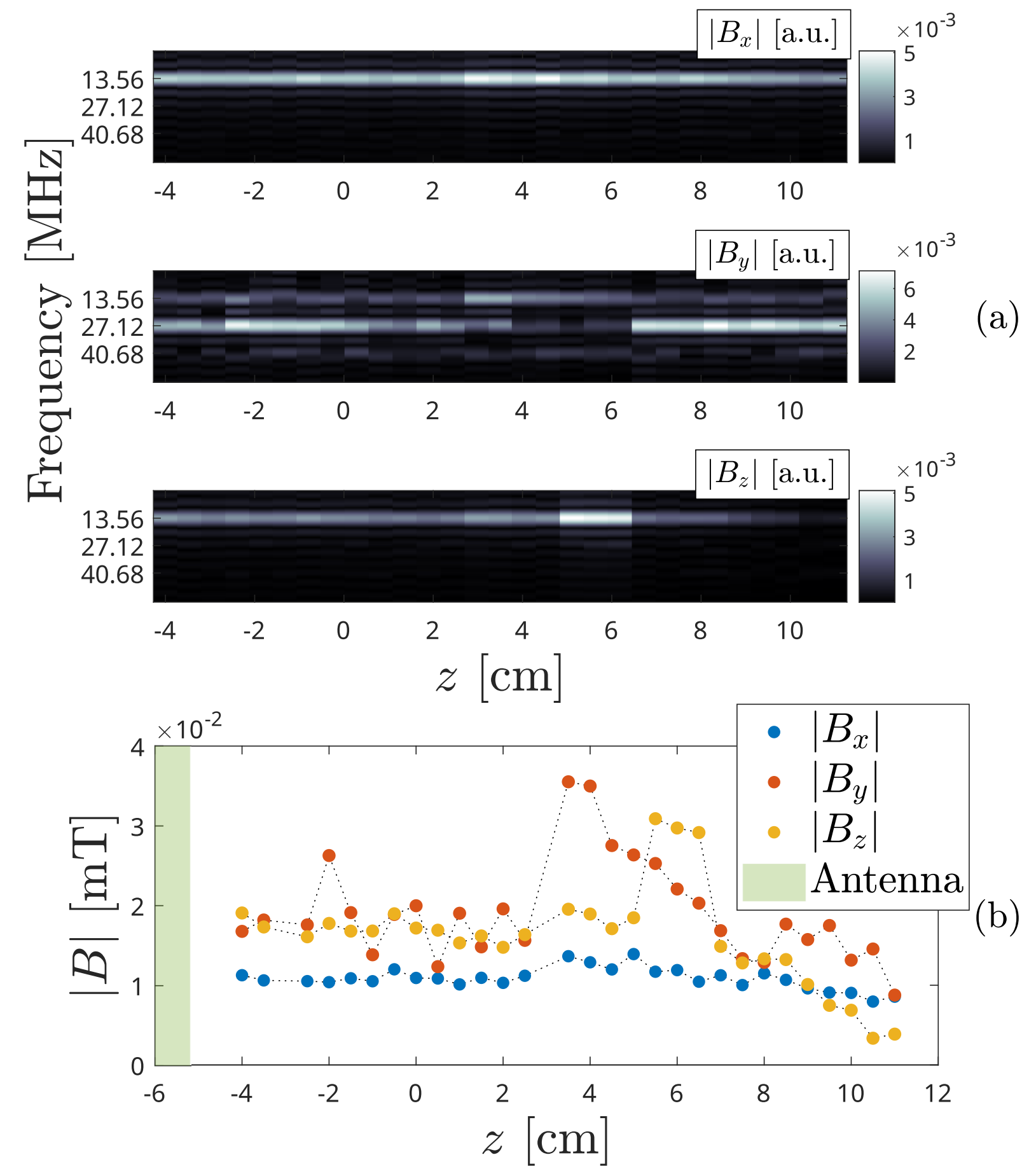




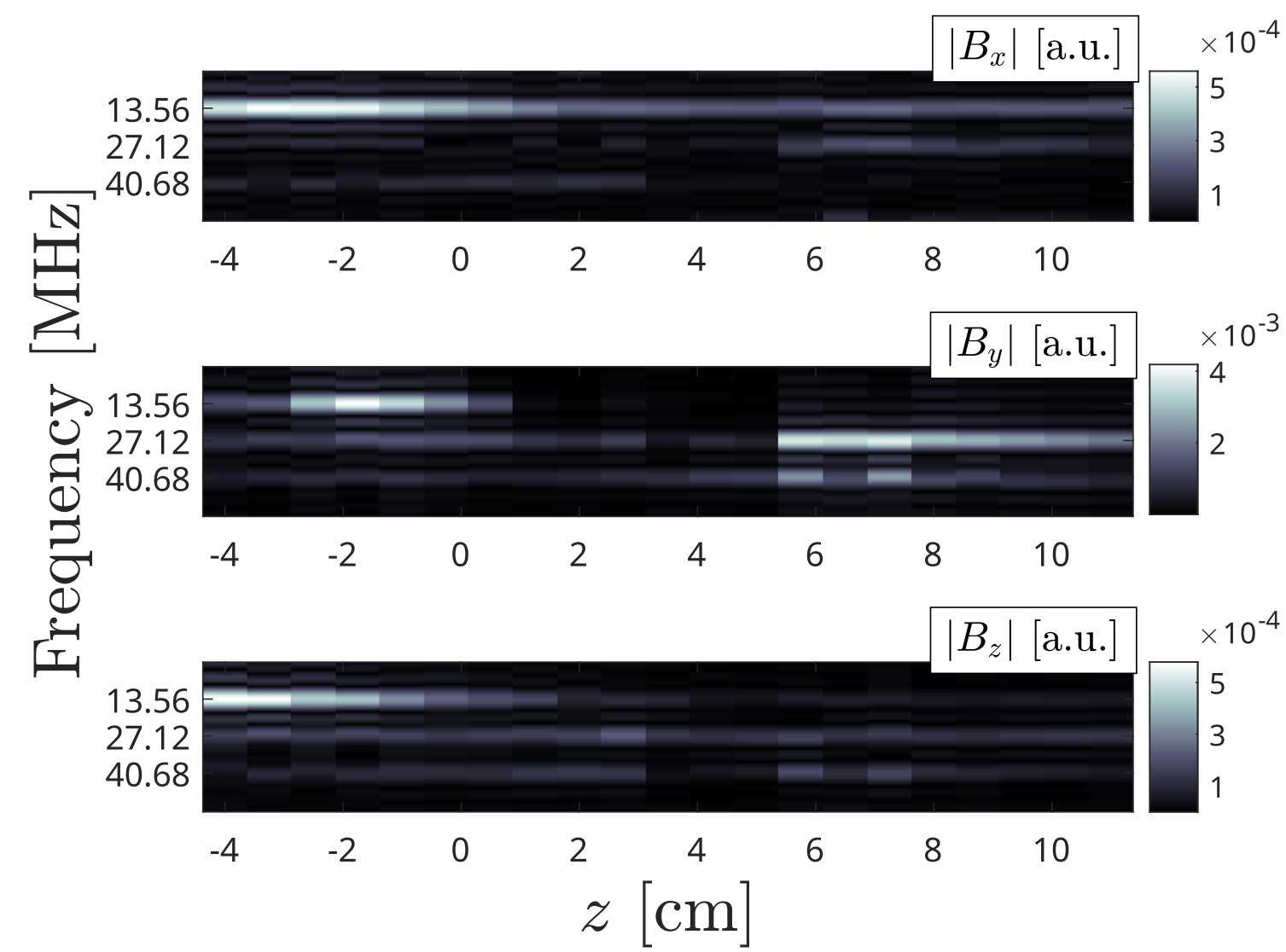

(a)

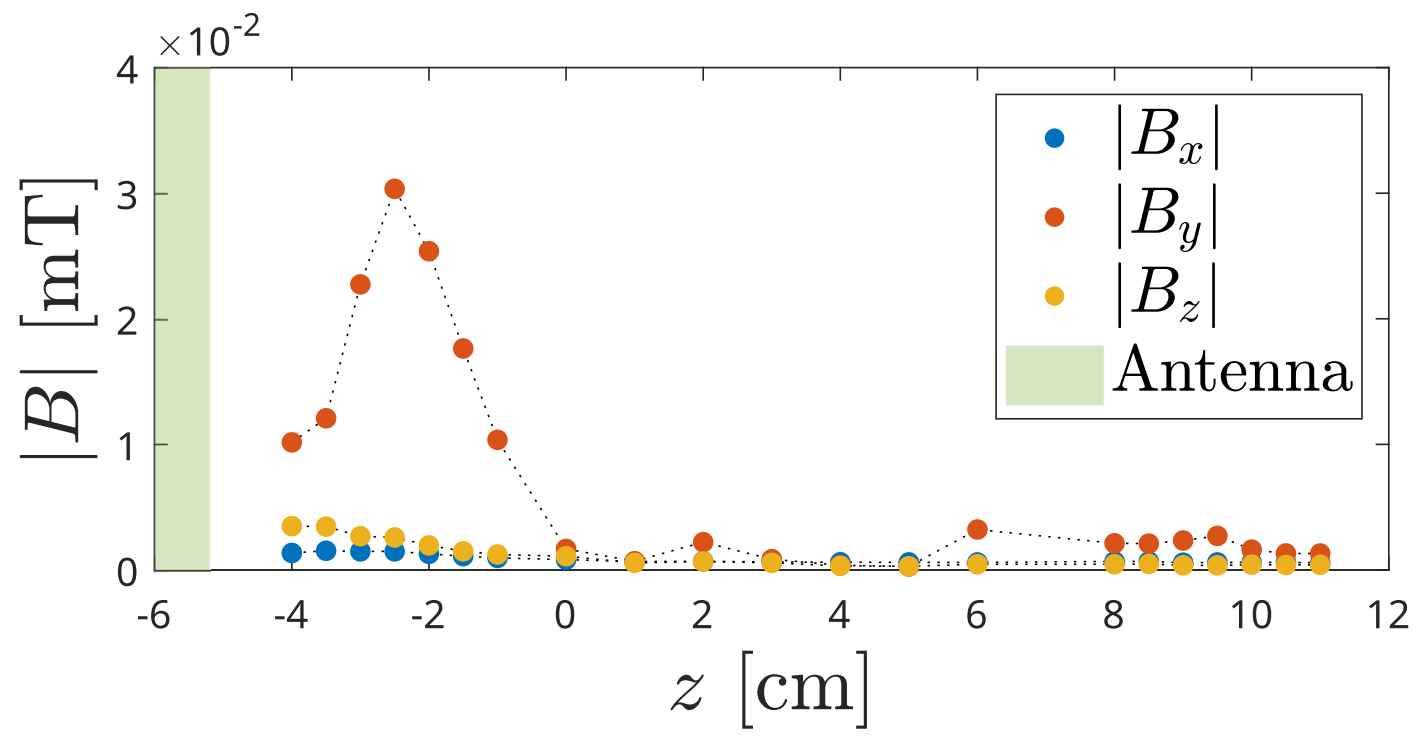

(b) 


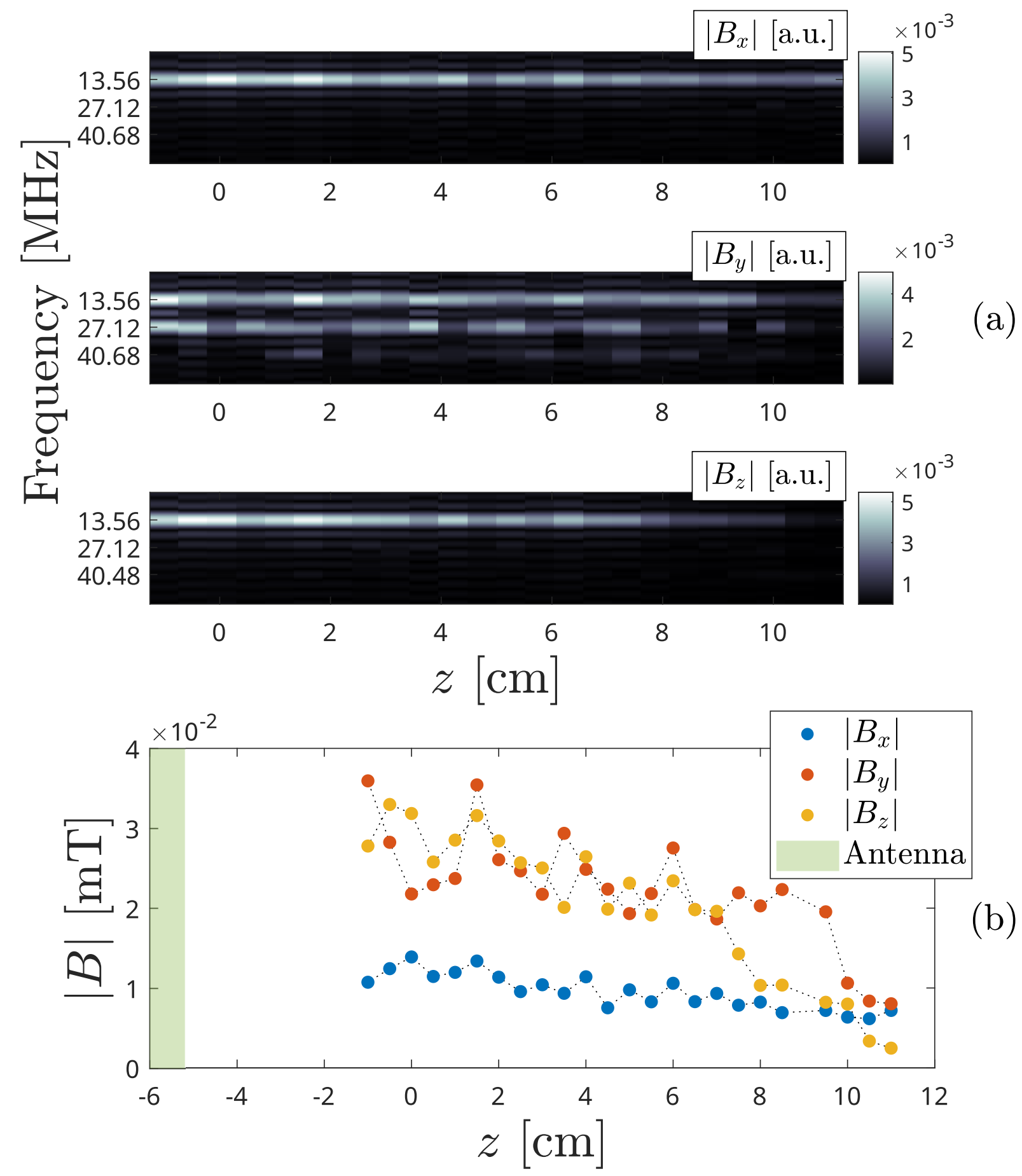




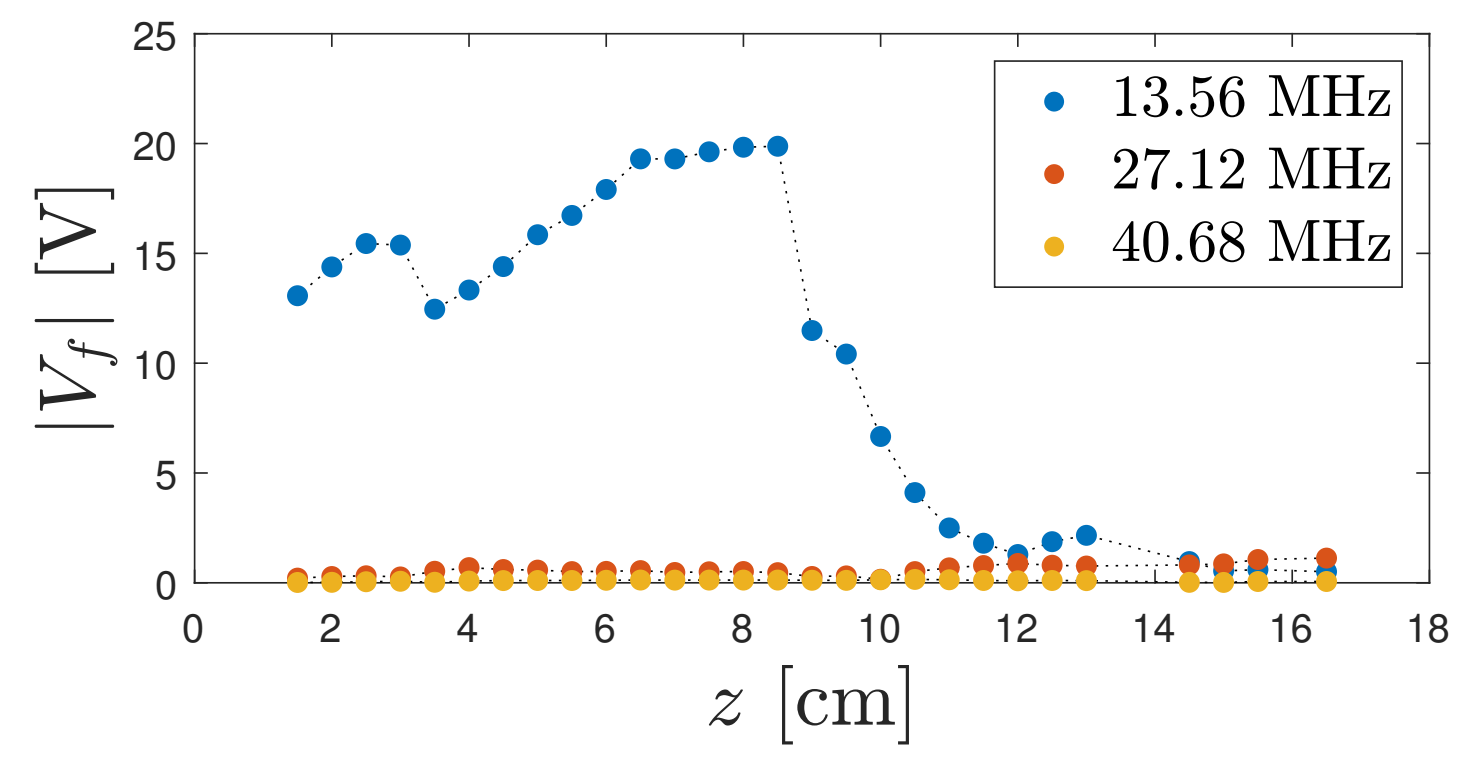




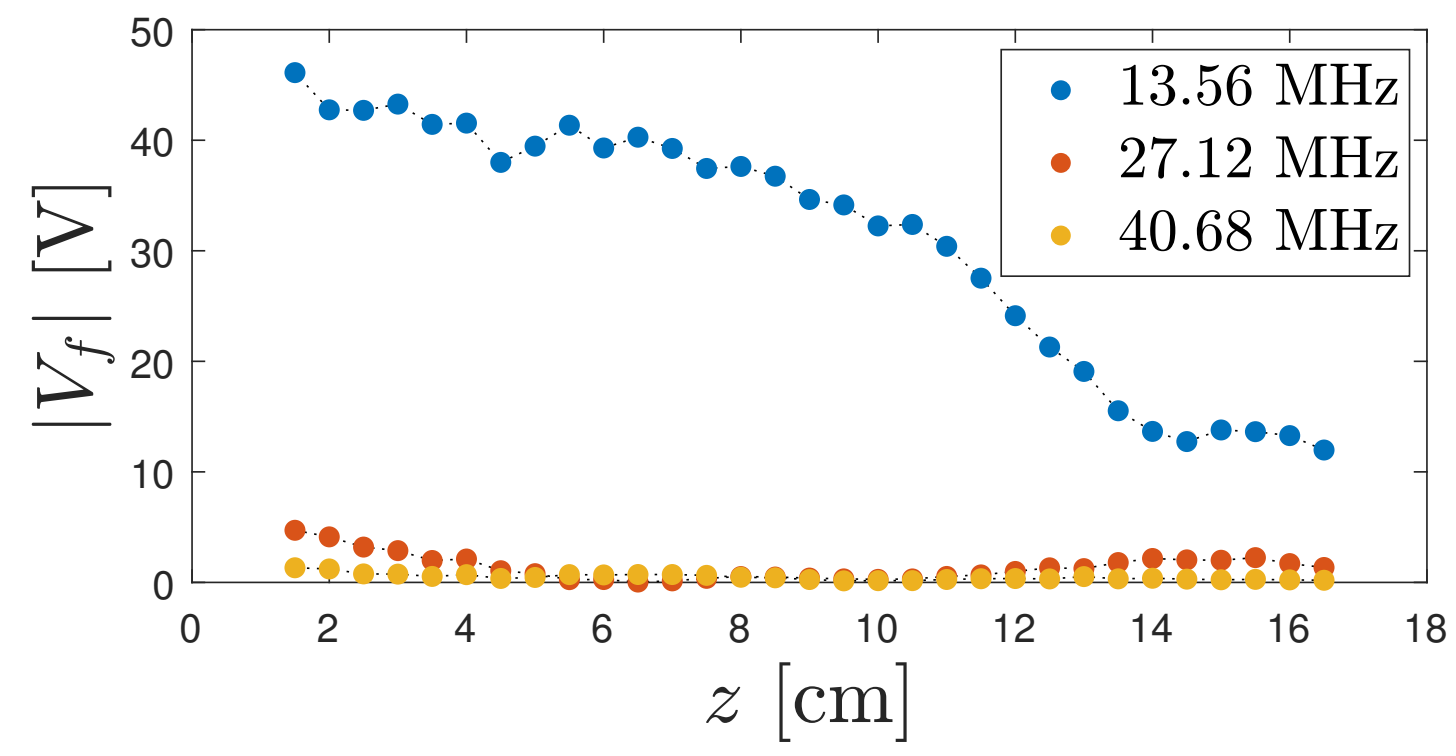




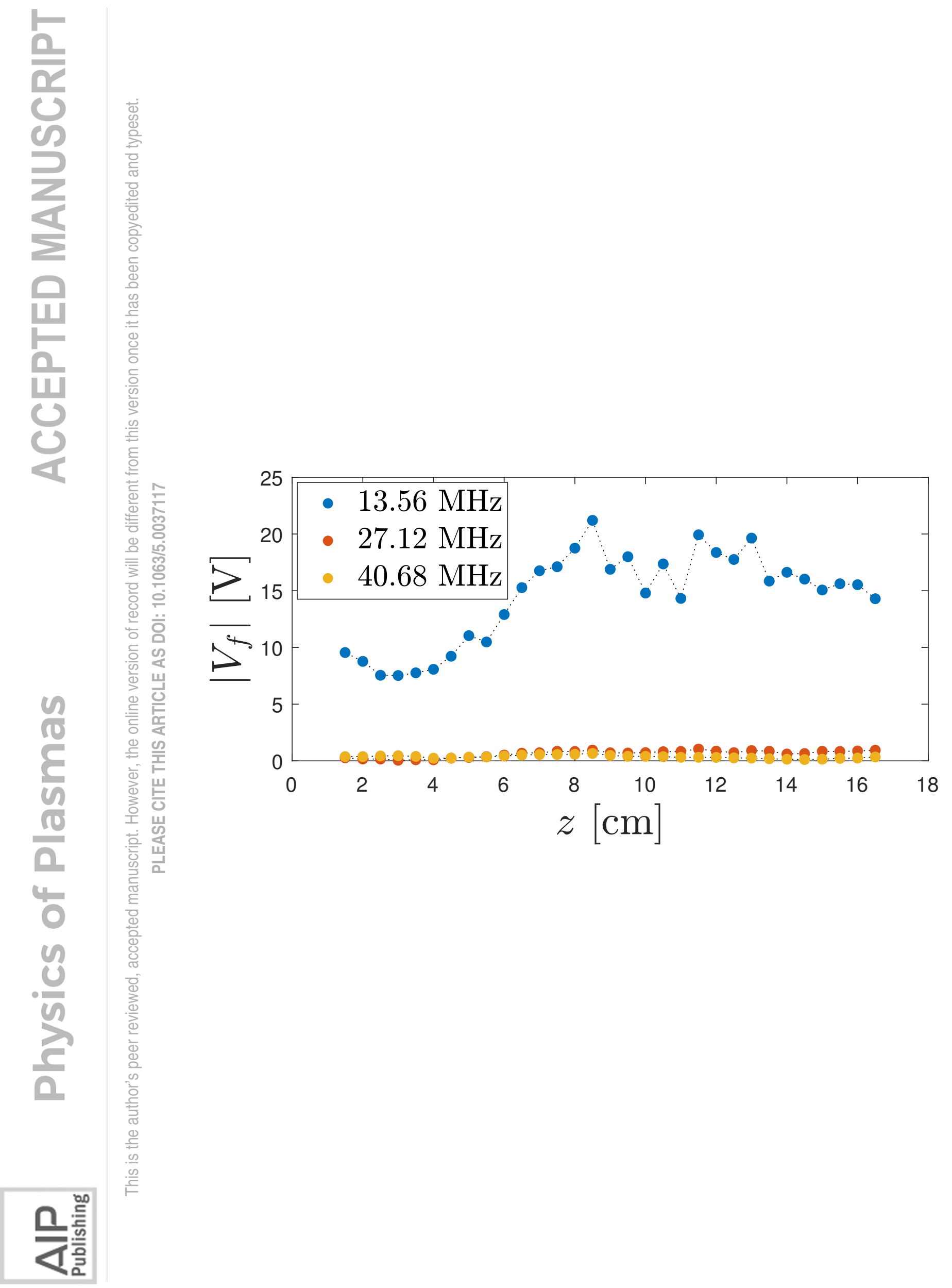




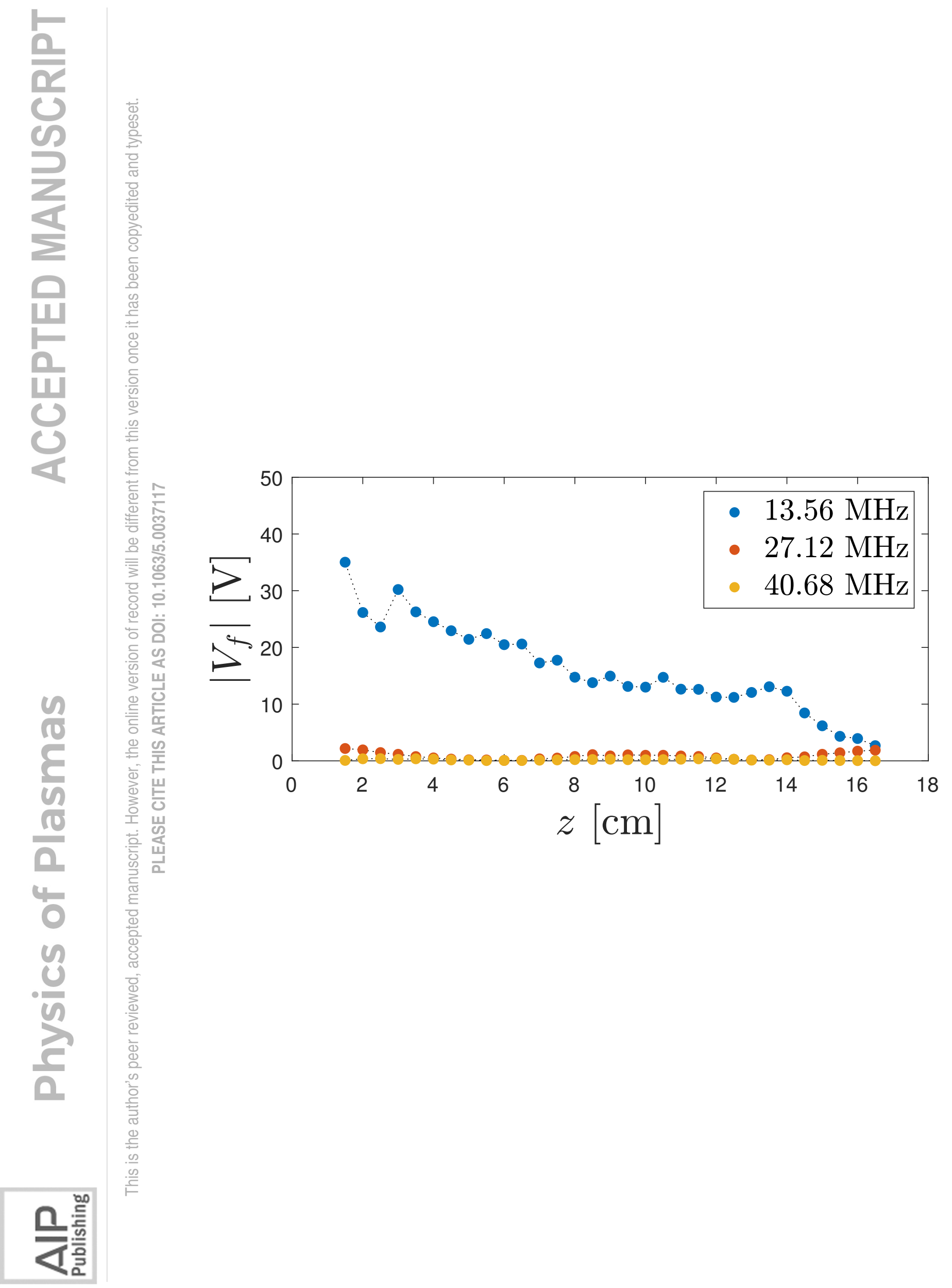



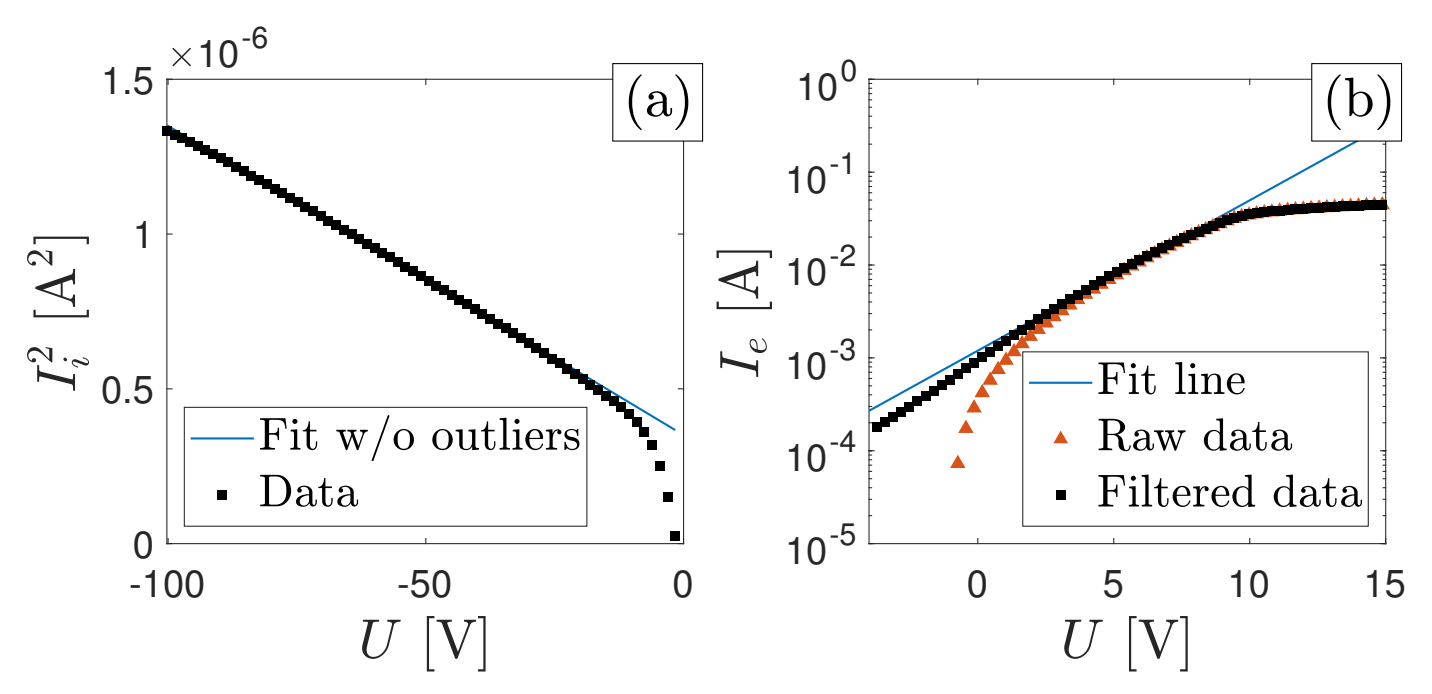

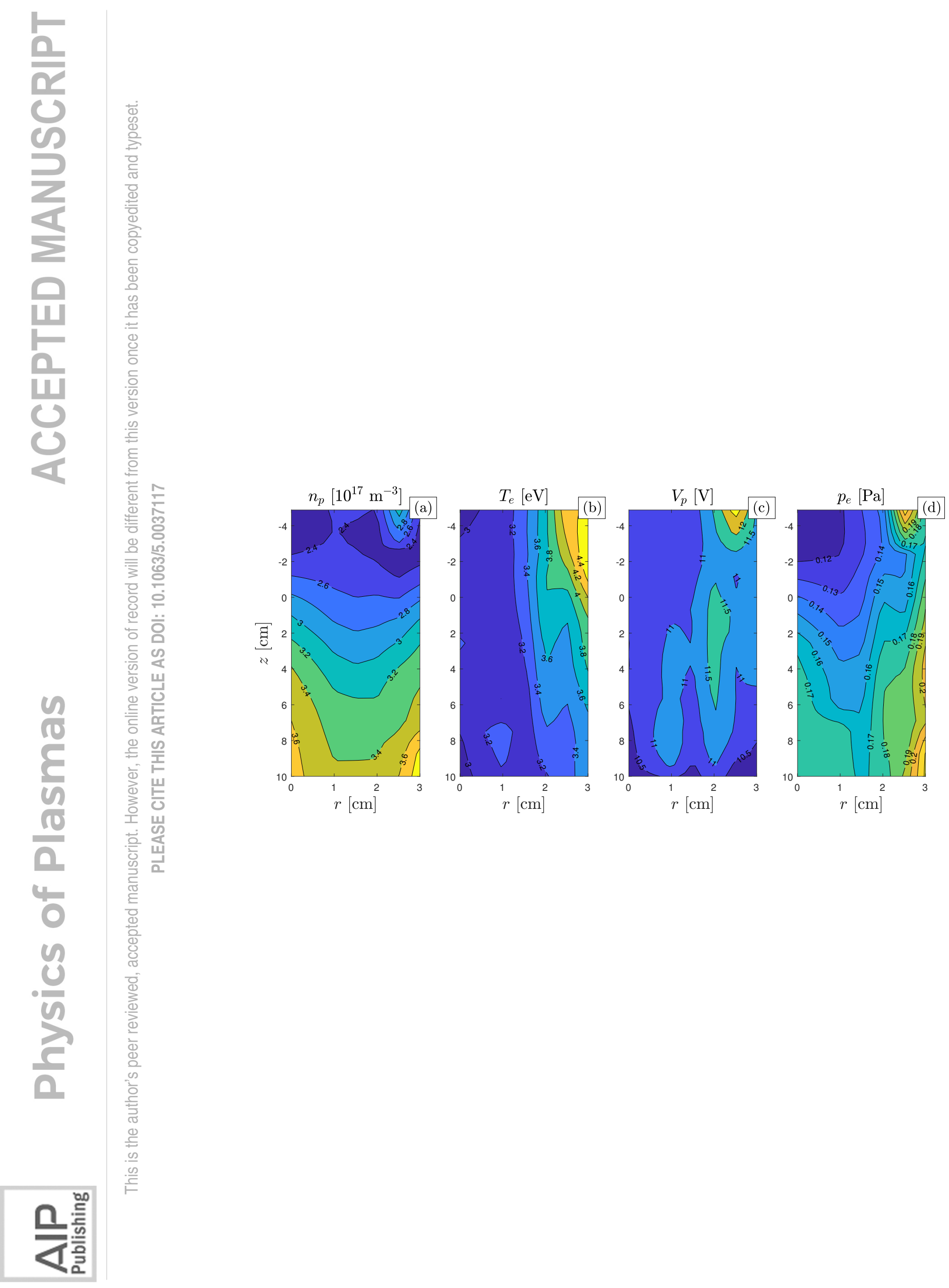

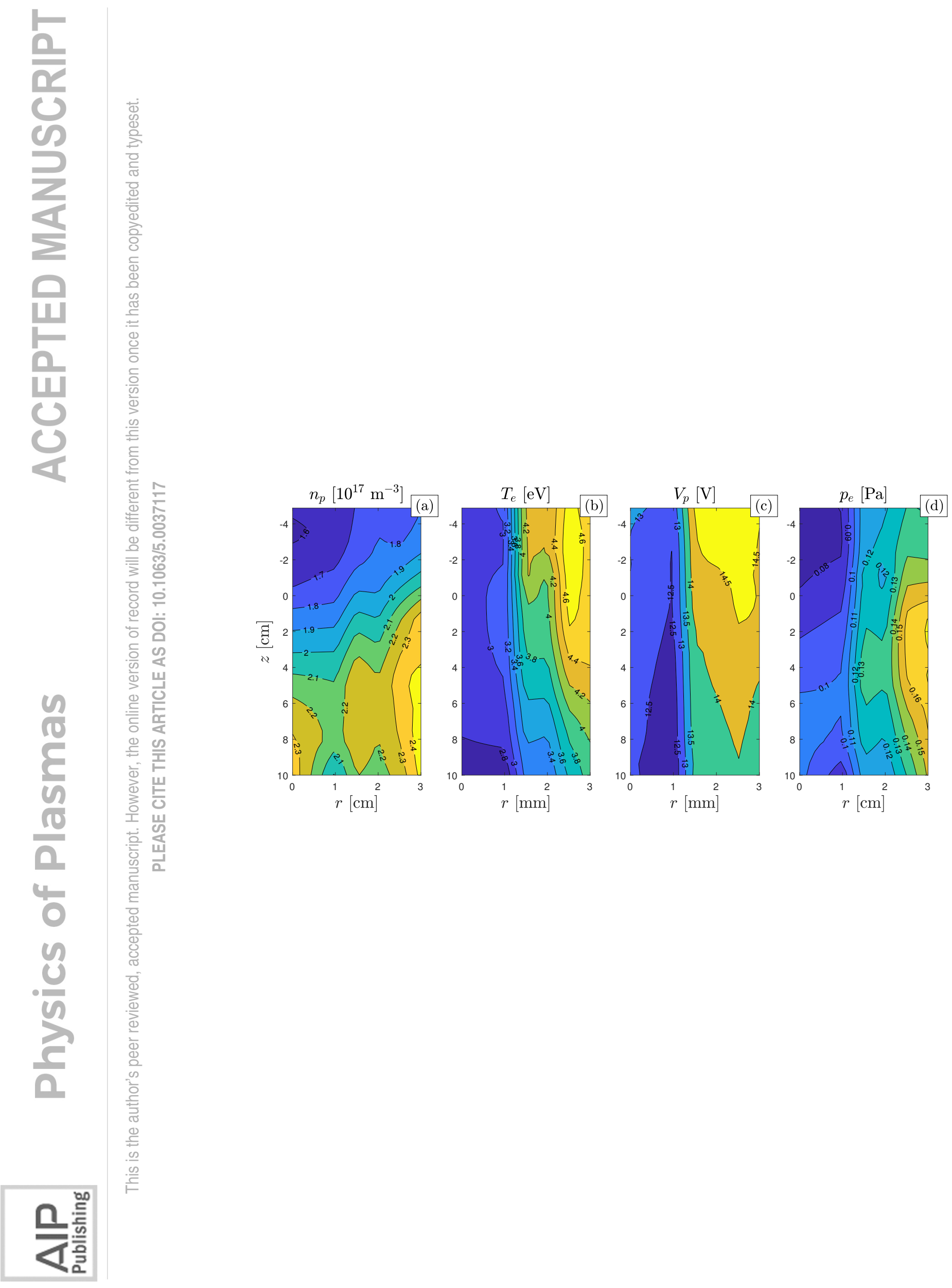


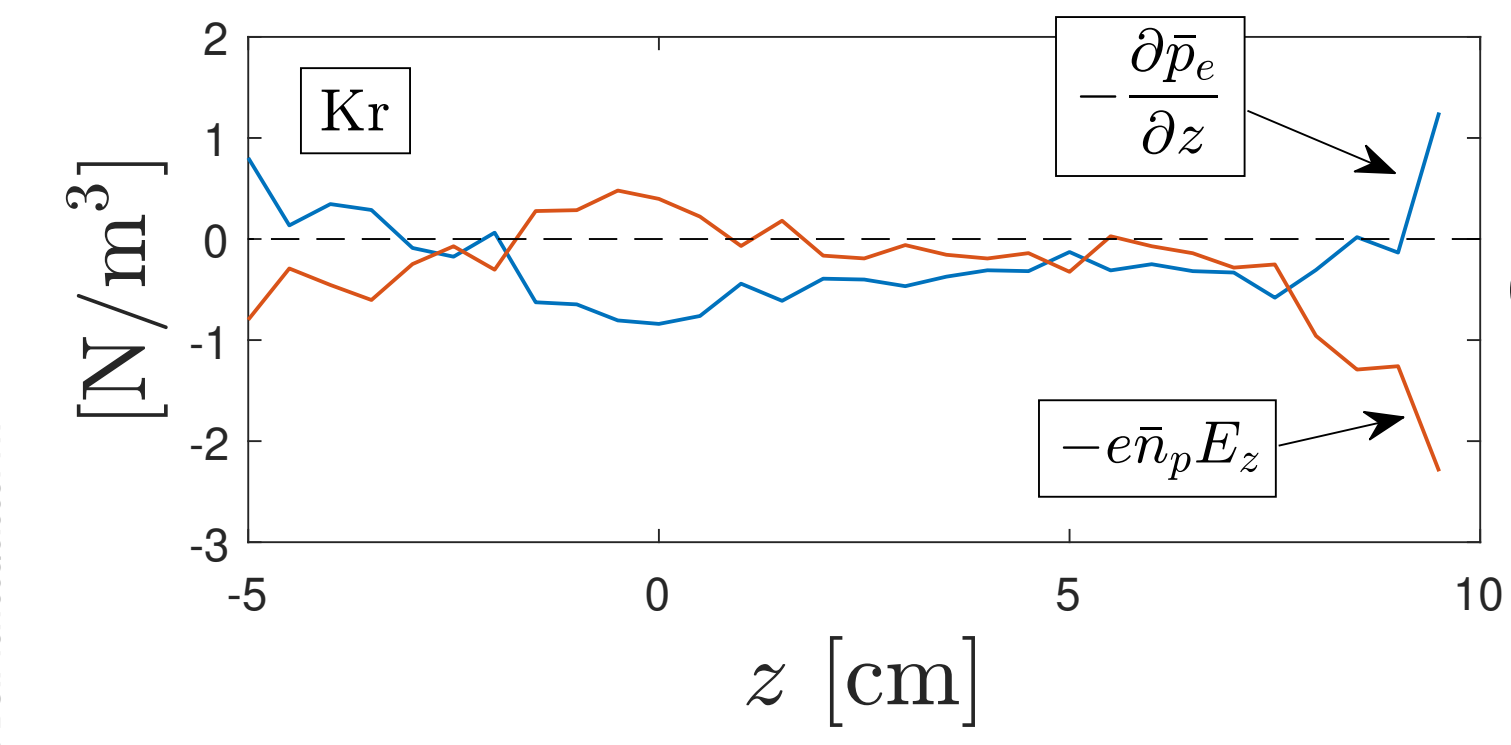

(a)

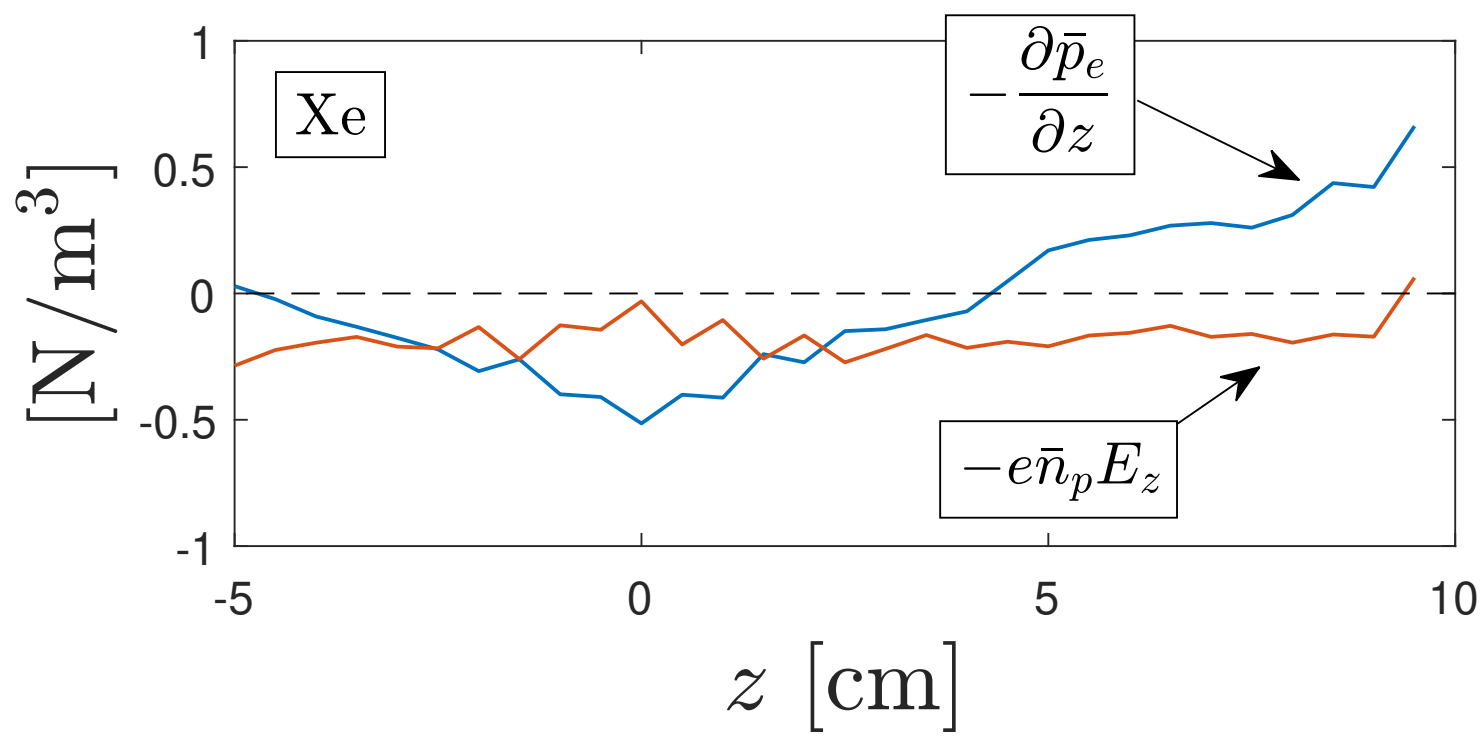

(b) 


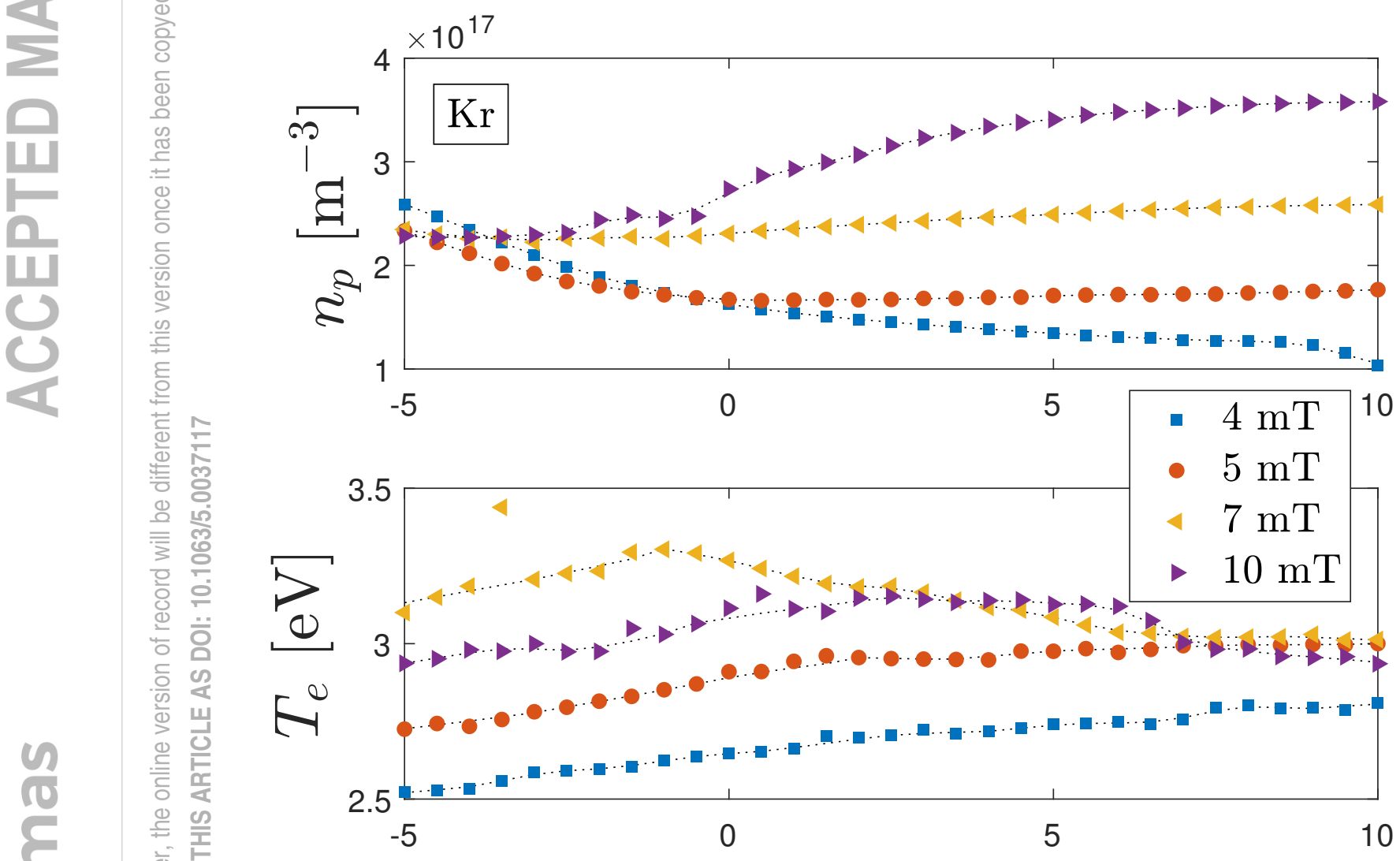

(a)

(b)

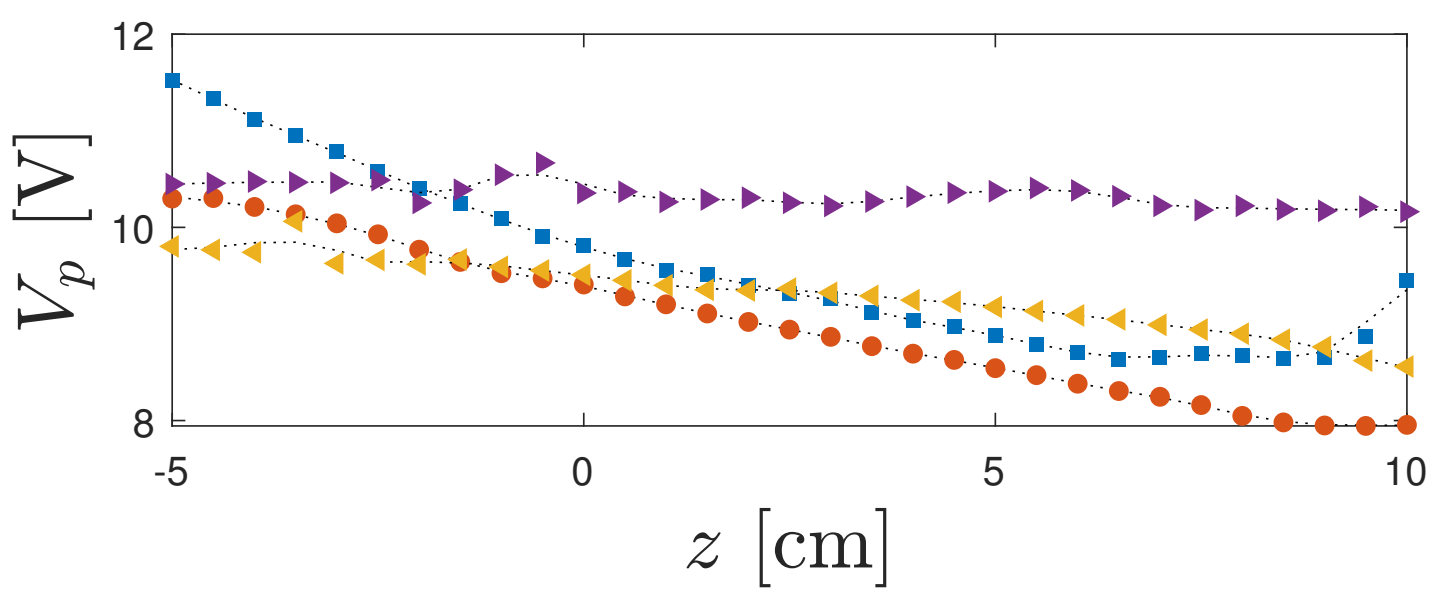




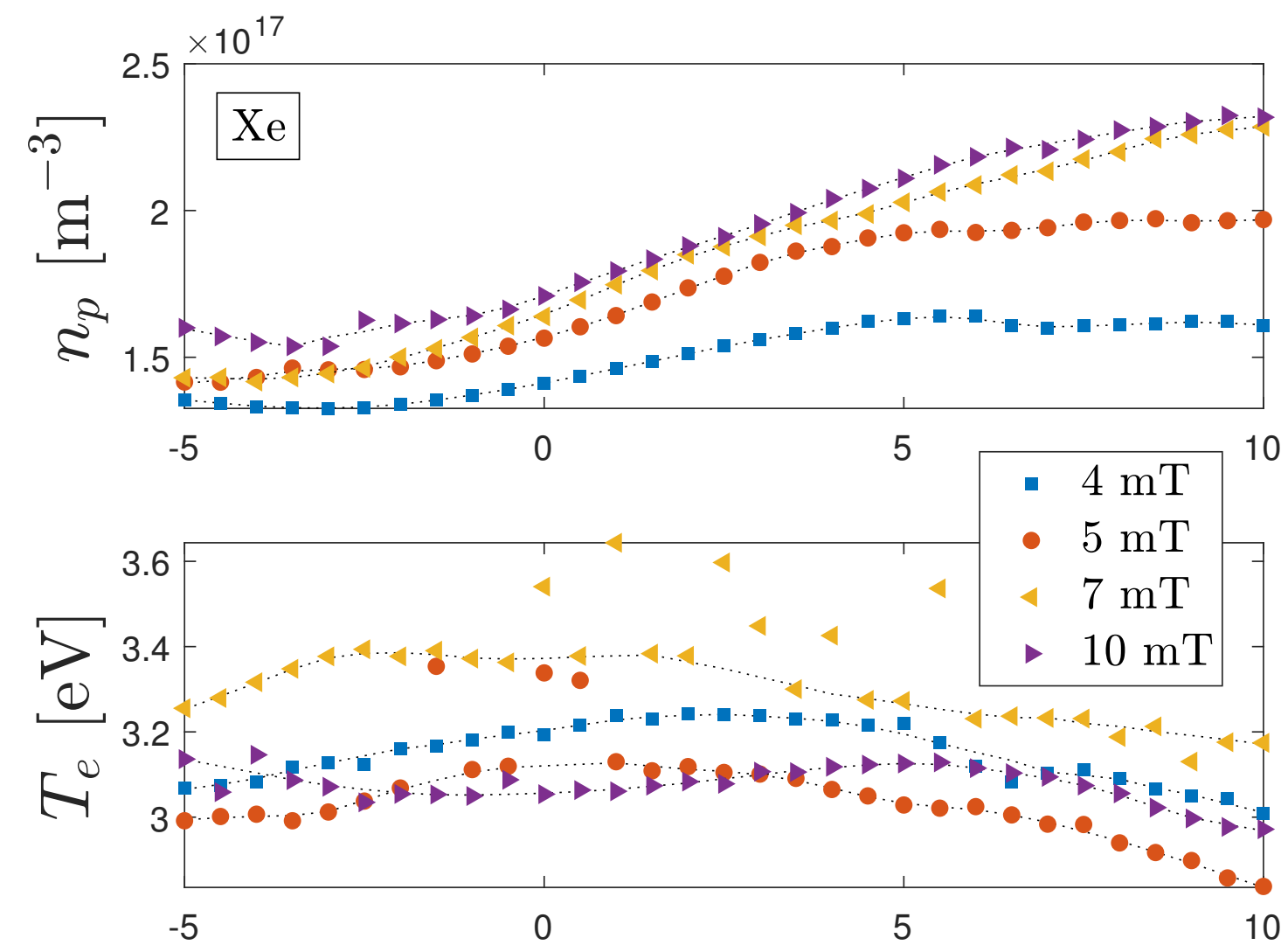

(a)

(b)

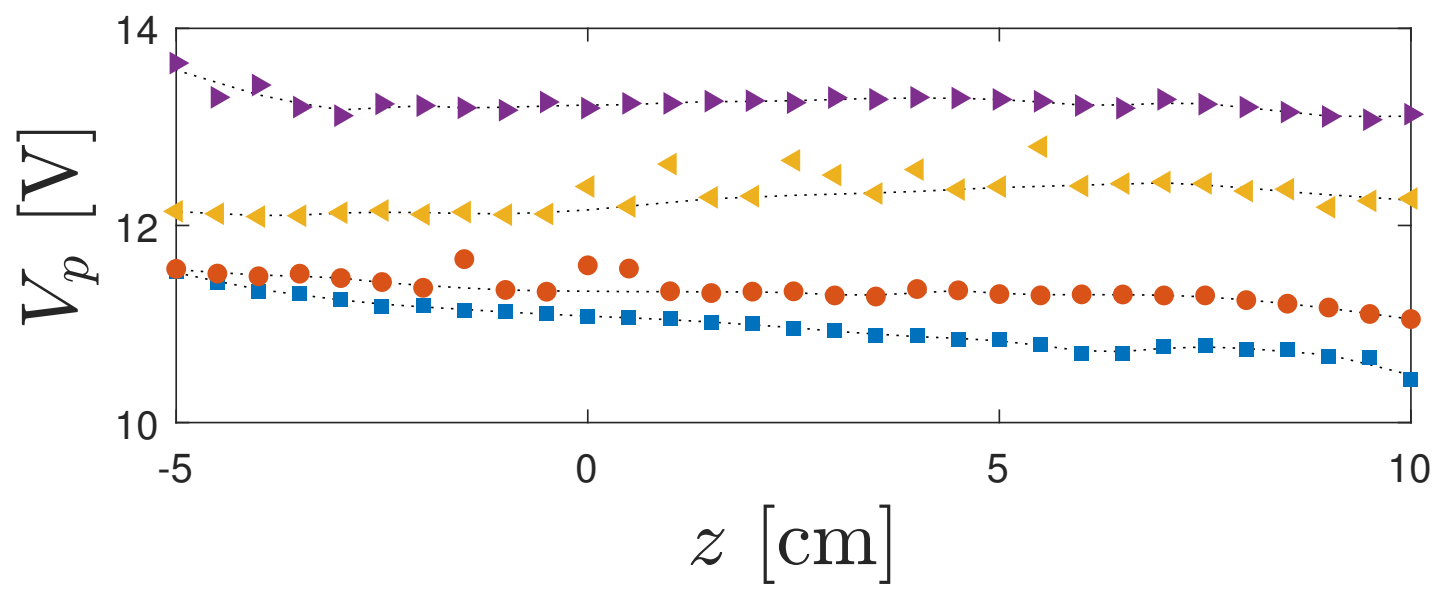

(c) 


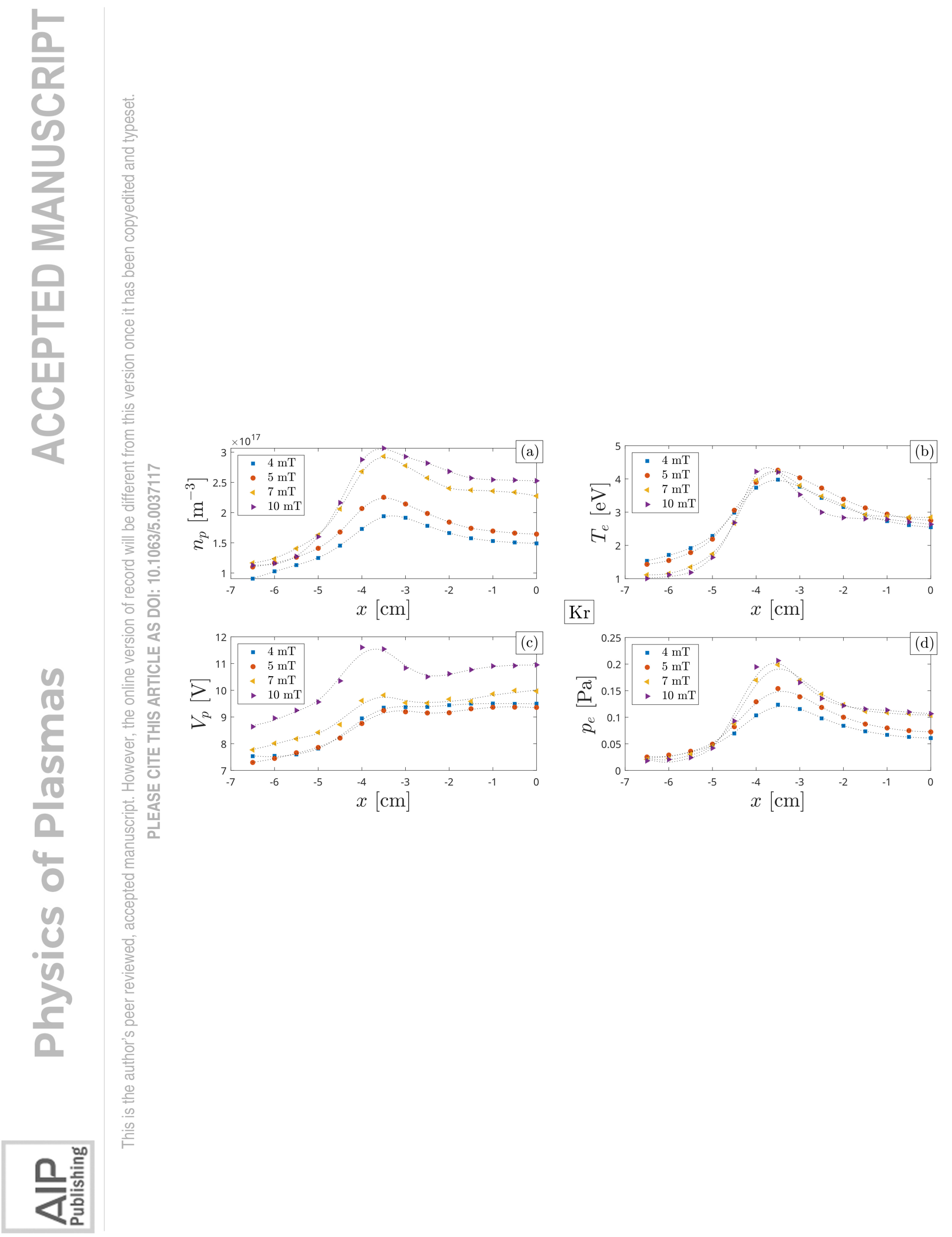




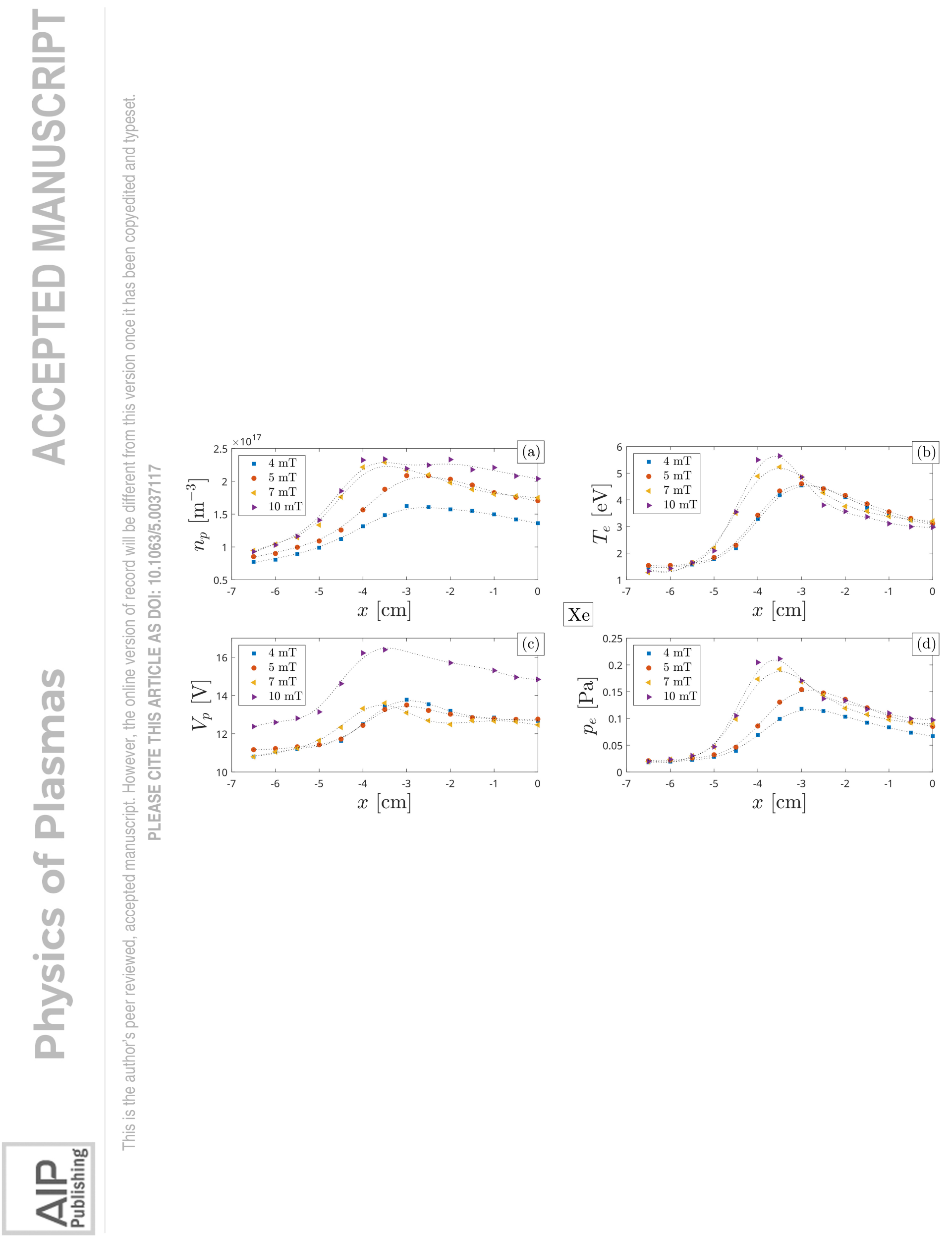




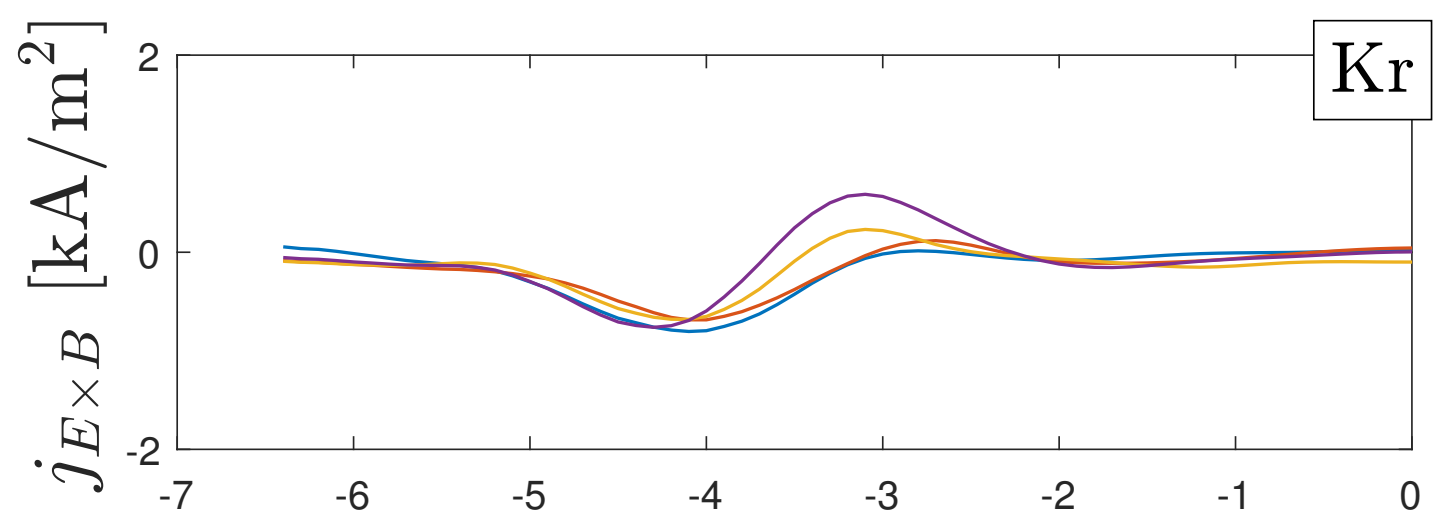

(a)

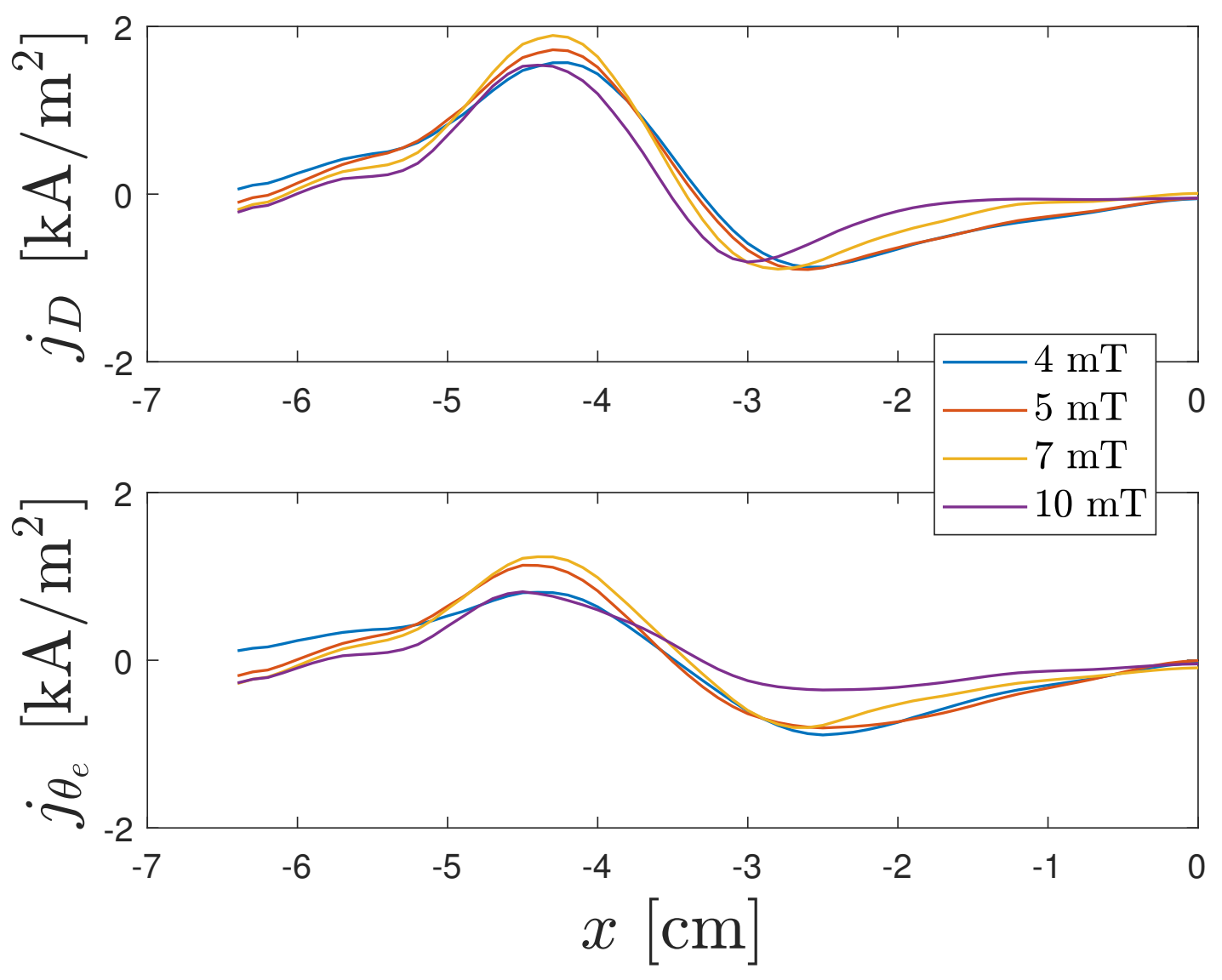

(b)

(c) 


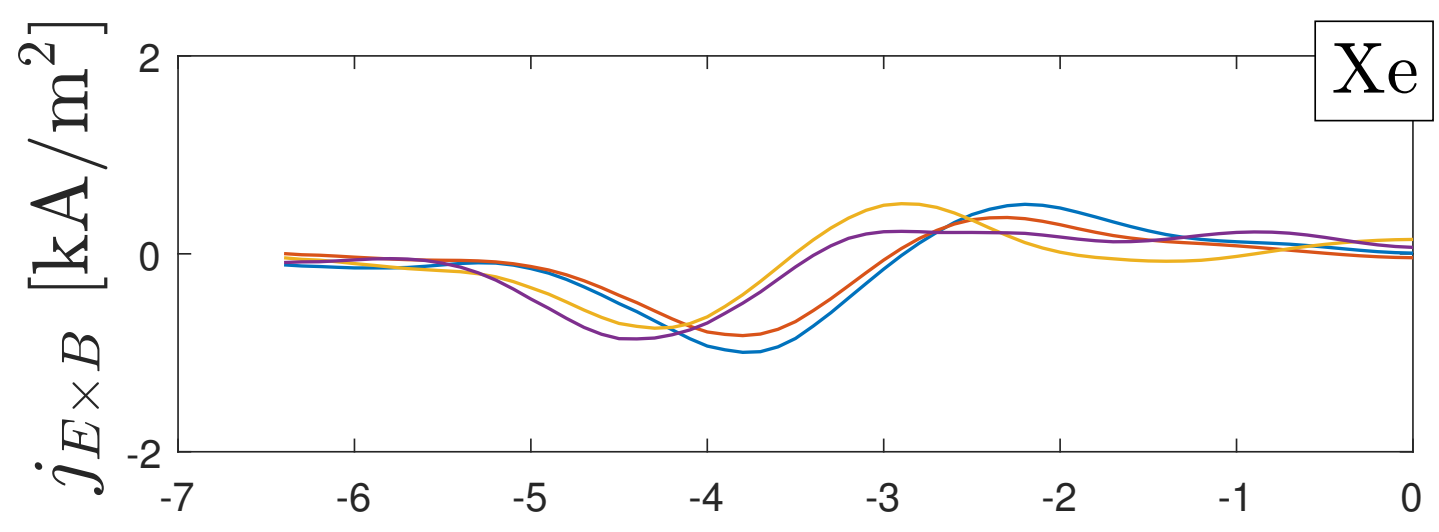

(a)

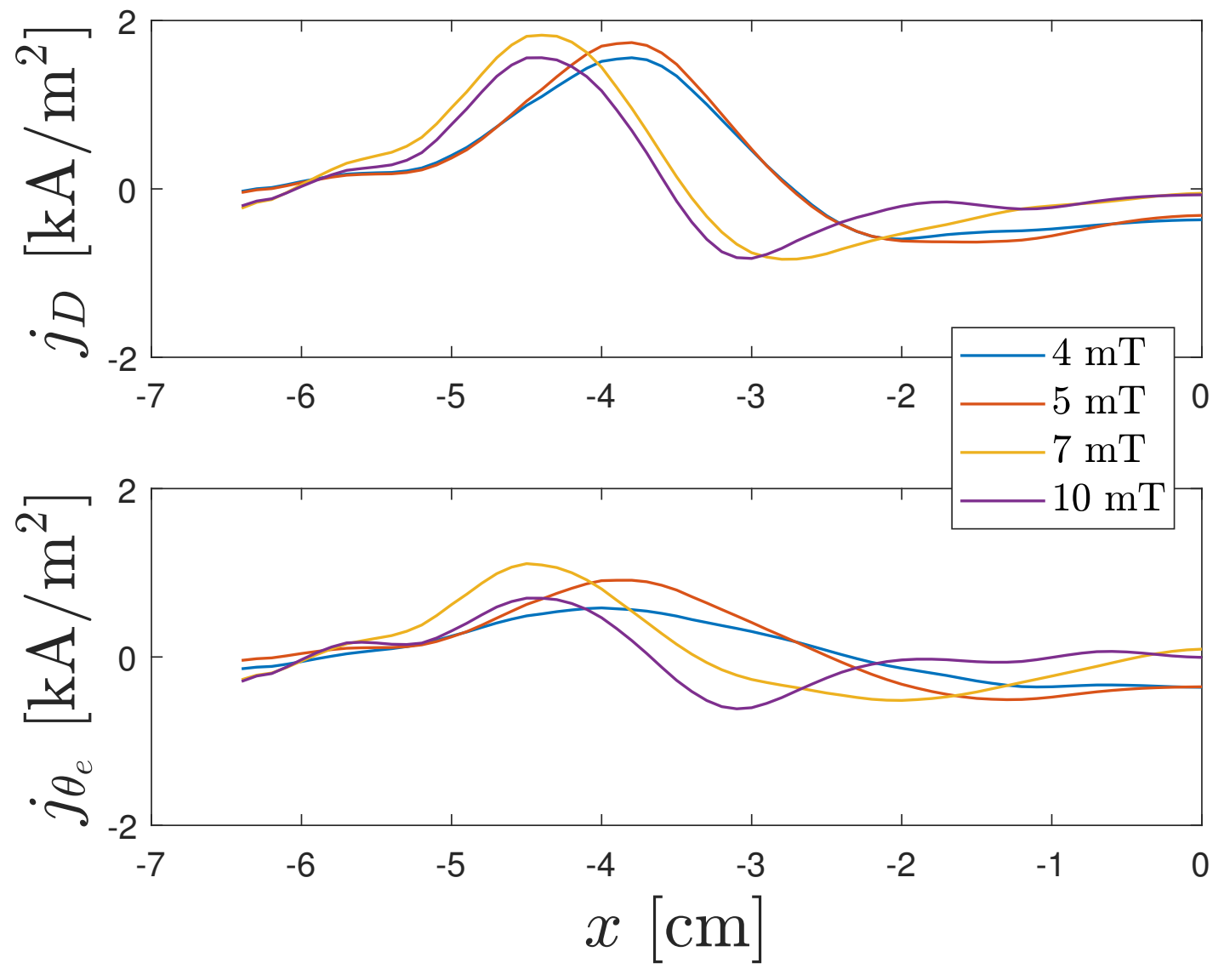

(b)

(c) 


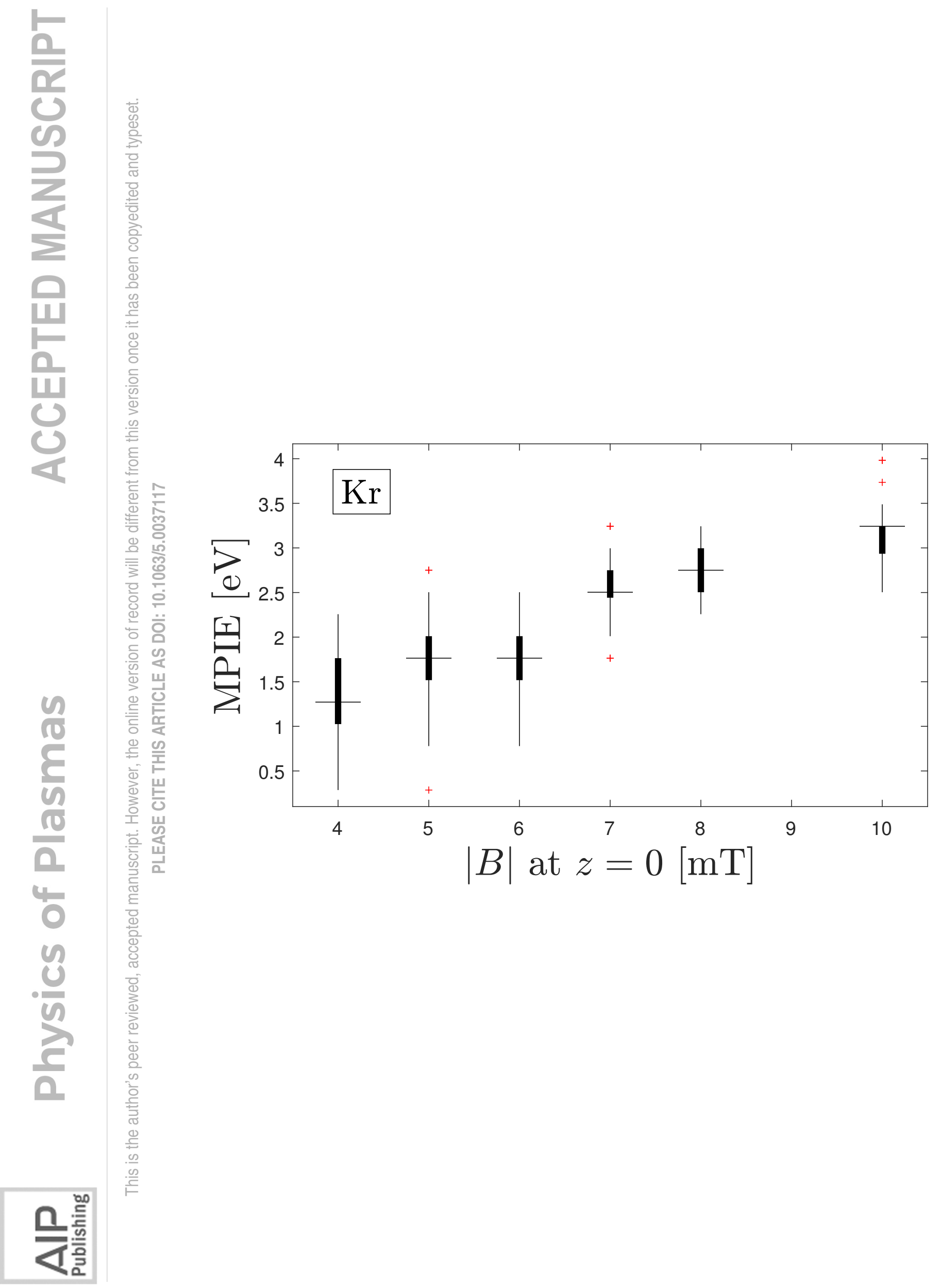




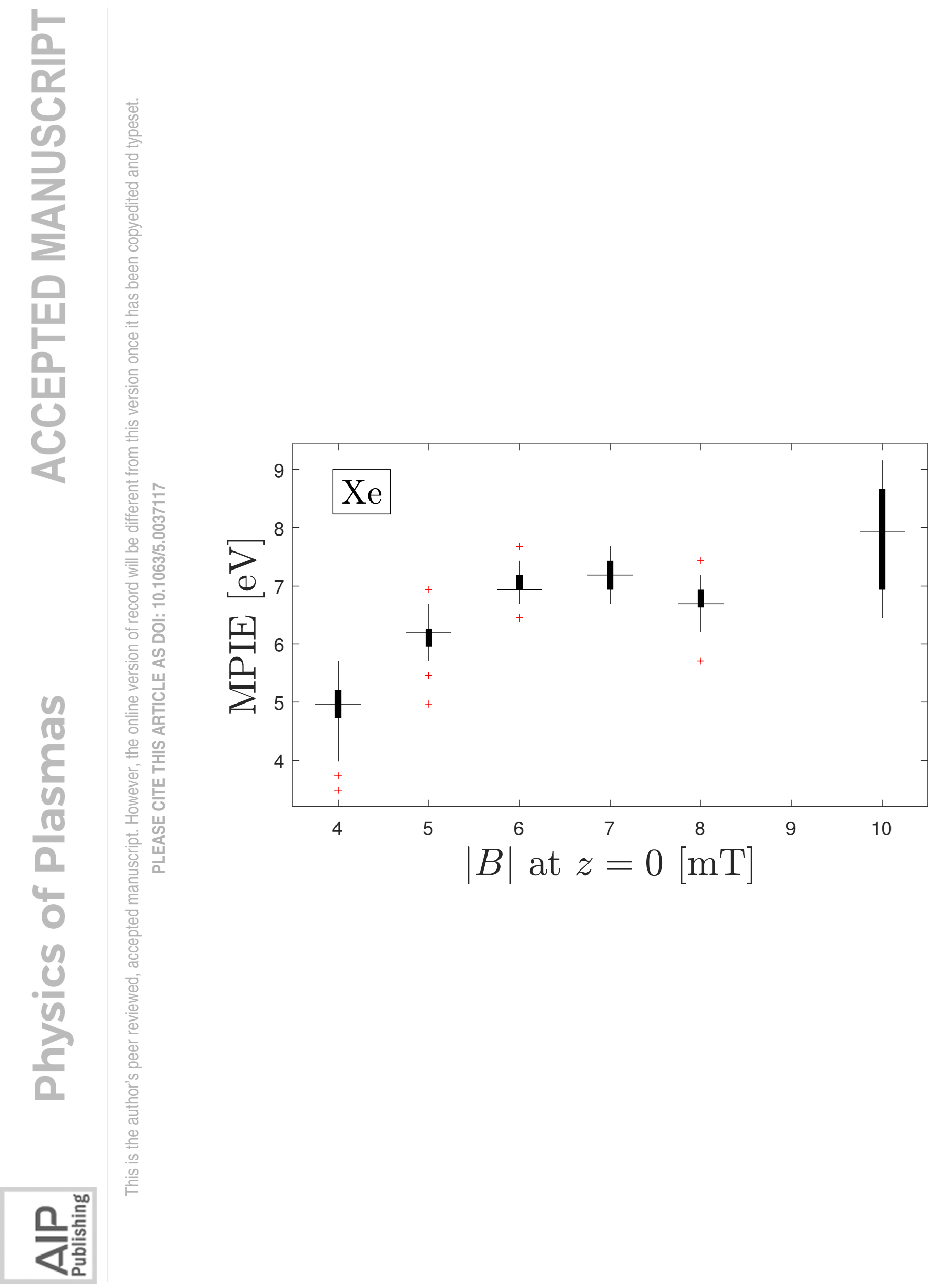

